\title{
ON THE VANISHING RANGES FOR THE COHOMOLOGY OF FINITE GROUPS OF LIE TYPE
}

\author{
CHRISTOPHER P. BENDEL, DANIEL K. NAKANO, AND CORNELIUS PILLEN
}

\begin{abstract}
Let $G\left(\mathbb{F}_{q}\right)$ be a finite Chevalley group defined over the field of $q=p^{r}$ elements, and $k$ be an algebraically closed field of characteristic $p>0$. A fundamental open and elusive problem has been the computation of the cohomology ring $\mathrm{H}^{\bullet}\left(G\left(\mathbb{F}_{q}\right), k\right)$. In this paper we determine initial vanishing ranges which improves upon known results. For root systems of type $A_{n}$ and $C_{n}$, the first non-trivial cohomology classes are determined when $p$ is larger than the Coxeter number (larger than twice the Coxeter number for type $A_{n}$ with $n>1$ and $r>1$ ). In the process we make use of techniques involving line bundle cohomology for the flag variety $G / B$ and its relation to combinatorial data from Kostant Partition Functions.
\end{abstract}

\section{Introduction}

1.1 Let $G$ be a simple algebraic group over an algebraically closed field $k$ of prime characteristic $p>0$ which is split over the prime field $\mathbb{F}_{p}$. Let $\mathrm{Fr}: G \rightarrow G$ denote the Frobenius map and set $q=p^{r}$. The fixed points of the $r$ th iterate of the Frobenius map, denoted $G\left(\mathbb{F}_{q}\right)$, is a finite Chevalley group. An outstanding open problem of major interest for algebraists and topologists has been to determine the cohomology $\operatorname{ring} \mathrm{H}^{\bullet}\left(G\left(\mathbb{F}_{q}\right), k\right)$. In 2005, during a talk at an Oberwolfach conference, Friedlander mentioned that so little is known about this computation that it is not even known in which degree the first non-trivial cohomology class occurs.

Our paper aims to address this fundamental question by investigating two problems:

(1.1.1) Determining Vanishing Ranges: Finding $D>0$ such that the cohomology groups $\mathrm{H}^{i}\left(G\left(\mathbb{F}_{q}\right), k\right)=0$ for $0<i<D$.

(1.1.2) Locating the First Non-Trivial Cohomology Class: In many instances in conjunction with the aforementioned problem, we will find a $D$ such that $\mathrm{H}^{i}\left(G\left(\mathbb{F}_{q}\right), k\right)=0$ for $0<i<D$ and $\mathrm{H}^{D}\left(G\left(\mathbb{F}_{q}\right), k\right) \neq 0$. A $D$ satisfying this property will be called a sharp bound.

There have been earlier results in the 1970s and 80s addressing (1.1.1). Quillen Q showed that $\mathrm{H}^{i}\left(G L_{n}\left(\mathbb{F}_{q}\right), k\right)=0$ for all $0<i<r(p-1)$ and all $n$. In that work, he noted

Date: June 2010.

Research of the first author was supported in part by NSF grant DMS-0400558.

Research of the second author was supported in part by NSF grant DMS-0654169.

2000 Mathematics Subject Classification. Primary 20J06; Secondary 20G10.

${ }^{1}$ In the cross-characteristic situation (i.e., if char $k=l$ and $\operatorname{gcd}(l, p)=1$ ) much more is known about the cohomology of $\mathrm{H}^{\bullet}\left(G\left(\mathbb{F}_{q}\right), k\right.$ ) (cf. AM Chapter VII]). In fact in many cases in the non-describing characteristic the cohomology (including the ring structure) is completely determined. 
that the arguments showed for any $G$ as above, there exists a constant $C$ depending on the root system such that $\mathrm{H}^{i}\left(G\left(\mathbb{F}_{q}\right), k\right)=0$ for $0<i<r \cdot C$. However, no explicit value of $C$ is given except for $G=S L_{2}$ (and $p$ odd) in which case one can take $C=(p-1) / 2$. Furthermore, it was not determined whether these vanishing ranges were sharp. Indeed, in the case of $S L_{2}$, one can see from work of Carlson [C] that these bounds are not sharp in general. Quillen's original work arose in the context of certain $K$-theory computations. Friedlander [F] later used $K$-theoretic techniques to find vanishing ranges for more general reductive groups. Later work of Hiller $[\mathrm{H}]$ extended Friedlander's result and found vanishing ranges for groups of all types.

Friedlander and Parshall [FP, (A.1) Lemma] found a sharp bound for the Borel subgroup $B\left(\mathbb{F}_{q}\right)$ of $G L_{n}\left(\mathbb{F}_{q}\right)$. Independent of this work, Barbu [B] constructed a non-zero cohomology class in $\mathrm{H}^{2 p-2}\left(G L_{n}\left(\mathbb{F}_{p}\right), k\right)$ for $p \geq n$. In this paper he conjectured that the sharp bound is $D=2 p-3$ for $G L_{n}\left(\mathbb{F}_{p}\right)$ when $n \geq 2$ and $p \geq 3$ (cf. [B. Section 1, Conjecture 4.11]). Since that time, few if any results have been obtained in this direction.

1.2 The strategy in addressing (1.1.1) and (1.1.2) will entail using new and powerful techniques developed by the authors which relate $\mathrm{H}^{i}\left(G\left(\mathbb{F}_{q}\right), k\right)$ to extensions over $G$ via a truncated version of the induction functor (cf. [BNP1, BNP2, BNP3, BNP5, BNP6]). An outline of the overall strategy is presented in the diagram below. For the purposes in this paper we will use a non-truncated induction functor $\mathcal{G}_{r}(-)$. We demonstrate that when applied to the trivial module $k, \mathcal{G}_{r}(k)$ has a filtration with factors of the form $H^{0}(\lambda) \otimes$ $H^{0}\left(\lambda^{*}\right)^{(r)}$ (cf. Proposition 2.4). The $G$-cohomology of these factors can be analyzed by using the Lyndon-Hochschild-Serre (LHS) spectral sequence involving the first Frobenius kernel $G_{r}$ (cf. Section 3.1). In particular for $r=1$, we can apply the results of Kumar-LauritzenThomsen [KLT] to bound the dimension of the cohomology group $\mathrm{H}^{\bullet}\left(G\left(\mathbb{F}_{p}\right), k\right)$ from above (cf. Theorem 3.3). The upper bound on the dimension involves the combinatorics of the well-studied Kostant Partition Function. This reduces the question of the vanishing of the finite group cohomology to a question involving the combinatorics of the underlying root system $\Phi$.

$$
\begin{aligned}
& \text { Induction } \\
& \text { Functor } \\
& \mathrm{H}^{i}\left(G\left(\mathbb{F}_{q}\right), k\right) \quad \Longrightarrow \quad \mathrm{H}^{i}\left(G, \mathcal{G}_{r}(k)\right) \\
& \Downarrow \quad \text { Filtrations } \\
& \mathrm{H}^{i}\left(G, H^{0}(\lambda) \otimes H\left(\lambda^{*}\right)^{(r)}\right) \quad \Longrightarrow \mathrm{H}^{i}\left(G_{1}, H^{0}(\lambda)\right) \quad \Longrightarrow \text { Root Combinatorics. } \\
& \text { LHS Spectral Kostant Partition } \\
& \text { Sequences Functions }
\end{aligned}
$$

More specifically, for a group $G$ of classical type and $\mathrm{H}^{i}\left(G\left(\mathbb{F}_{q}\right), k\right)$, under the assumption that $p>h$ (the Coxeter number), in Theorem 4.4, we identify a vanishing range which 
improves upon (in almost all cases) the ranges of $\left[\mathrm{H}\right.$. Furthermore, in type $C_{n}$ and $A_{n}$, we identify a sharp vanishing bound which addresses (1.1.2) (cf. Theorems 5.4, 6.13, 6.14). These bounds are established for primes larger than the Coxeter number, except for type $A_{n}$ with $r>1$ where sharp vanishing bounds are found for $p$ greater than twice the Coxeter number. Finally, as a demonstration of the effectiveness of our methods we verify Barbu's Conjecture for $G=G L_{n}\left(\mathbb{F}_{q}\right)$ when $n \geq 2$ and $p \geq n+2$ (cf. Theorem 6.15).

Our results provide a conceptual description of how the geometry of the nilpotent cone plays a role in the description of the cohomology $\mathrm{H}^{i}\left(G\left(\mathbb{F}_{p}\right), k\right)$. In particular we prove for $p>h$ (cf. Theorem 3.3):

$$
\operatorname{dim} H^{i}\left(G\left(\mathbb{F}_{p}\right), k\right) \leq \sum_{\{w \in W \mid \ell(w) \equiv i \bmod 2\}} \sum_{\mu \in X(T)_{+}} \sum_{u \in W}(-1)^{\ell(u)} P_{\frac{i-\ell(w)}{2}}(u \cdot(p \mu+w \cdot 0)-\mu) .
$$

Here, $P_{n}(-)$ is the Kostant Partition Function. The root combinatorics involving the Kostant Partition Function naturally arises in the context of composition factor multiplicities in the ring of regular functions on the nilpotent cone $\mathcal{N}$ of $\mathfrak{g}=$ Lie $G$ (cf. [J2] $[\mathrm{Br}$ ). This result reinforces work of Carlson, Lin and Nakano [CLN] where they prove that the spectrum of this cohomology ring is given by the coordinate algebra on $\mathcal{N}^{\mathbb{F}} p / G\left(\mathbb{F}_{p}\right)$ where $\mathcal{N}^{\mathbb{F} p}$ is the variety inside of $\mathcal{N}$ consisting of $\mathbb{F}_{p^{-}}$expressible elements.

The sections of the paper are outlined as follows. In Section 2, we review our previous work and develop the necessary cohomological tools related to induction functors which will be used to determine vanishing ranges. In Section 3, we present some further cohomological properties relating extensions over $G$ with those over the Frobenius kernel $G_{r}$. In Section 4, our general vanishing bounds are presented. Finally, Sections 5 and 6 deal with the special cases of root systems of types $C_{n}$ and $A_{n}$ respectively. The reader might be surprised to see type $C$ treated prior to type $A$. It turns out that the root systems of type $C$ are by far the easiest to be dealt with. The authors in future work plan to address (1.1.1) and (1.1.2) in the case of the remaining classical groups and the exceptional groups.

\section{Relating $G\left(\mathbb{F}_{q}\right)$ and $G$}

2.1 Notation. Throughout this paper, we will follow the basic conventions provided in [J1. Let $G$ be a simple simply connected algebraic group scheme which is defined and split over the finite field $\mathbb{F}_{p}$ with $p$ elements, and let $k$ be a field of characteristic $p$. For $r \geq 1$, let $G_{r}:=\operatorname{ker} F^{r}$ be the $r$ th Frobenius kernel of $G$ and $G\left(\mathbb{F}_{q}\right)$ be the associated finite Chevalley group. Let $T$ be a maximal split torus and $\Phi$ be the root system associated to $(G, T)$. The positive (resp. negative) roots are $\Phi^{+}$(resp. $\Phi^{-}$), and $\Delta$ is the set of simple roots. Let $B$ be a Borel subgroup containing $T$ corresponding to the negative roots and $U$ be the unipotent radical of $B$. For a given root system of rank $n$, the simple roots will be denoted by $\alpha_{1}, \alpha_{2}, \ldots, \alpha_{n}$. We will adhere to the Bourbaki ordering of simple roots. In particular, for type $B_{n}, \alpha_{n}$ denotes the unique short simple root and for type $C_{n}, \alpha_{n}$ denotes the unique long simple root. The longest (positive) root will be denoted $\tilde{\alpha}$, and for root systems with multiple root lengths, the longest short root will be denoted $\alpha_{0}$. Let $W$ denote the Weyl group associated to $\Phi$, and, for $w \in W$, let $\ell(w)$ denote the length of the word. 
Let $\mathbb{E}$ be the Euclidean space associated with $\Phi$, and the inner product on $\mathbb{E}$ will be denoted by $\langle$,$\rangle . Let \alpha^{\vee}=2 \alpha /\langle\alpha, \alpha\rangle$ be the coroot corresponding to $\alpha \in \Phi$. In this case, the fundamental weights (basis dual to $\alpha_{1}^{\vee}, \alpha_{2}^{\vee}, \ldots, \alpha_{n}^{\vee}$ ) will be denoted by $\omega_{1}, \omega_{2}, \ldots, \omega_{n}$. Let $X(T)$ be the integral weight lattice spanned by these fundamental weights. The set of dominant integral weights is denoted by $X(T)_{+}$. For a weight $\lambda \in X(T)$, set $\lambda^{*}:=-w_{0} \lambda$ where $w_{0}$ is the longest word in the Weyl group $W$. By $w \cdot \lambda:=w(\lambda+\rho)-\rho$ we denote the "dot" action of $W$ on $X(T)$, with $\rho$ being the half-sum of the positive roots. For $\alpha \in \Delta$, $s_{\alpha} \in W$ denotes the reflection in the hyperplane determined by $\alpha$.

For a $G$-module $M$, let $M^{(r)}$ be the module obtained by composing the underlying representation for $M$ with $F^{r}$. Moreover, let $M^{*}$ denote the dual module. For $\lambda \in X(T)_{+}$, let $H^{0}(\lambda):=\operatorname{ind}_{B}^{G} \lambda$ be the induced module and $V(\lambda):=H^{0}\left(\lambda^{*}\right)^{*}$ be the Weyl module of highest weight $\lambda$.

2.2 We record two observations on roots that will be used at various points in the paper:

Observation (A). If $\beta \in \Phi^{+}$with $\beta \neq \tilde{\alpha}$, then $\left\langle\beta, \tilde{\alpha}^{\vee}\right\rangle \in\{0,1\}$.

Observation (B). If $w \in W$ admits a reduced expression $w=s_{\beta_{1}} s_{\beta_{2}} \ldots s_{\beta_{m}}$ with $\beta_{i} \in \Delta$ and $m=\ell(w)$, then

$$
-w \cdot 0=\beta_{1}+s_{\beta_{1}}\left(\beta_{2}\right)+s_{\beta_{1}} s_{\beta_{2}}\left(\beta_{3}\right)+\cdots+s_{\beta_{1}} s_{\beta_{2}} \ldots s_{\beta_{m-1}}\left(\beta_{m}\right) .
$$

Moreover, this is the unique way in which $-w \cdot 0$ can be expressed as a sum of distinct positive roots.

2.3 The Induction Functor. Set $\mathcal{G}_{r}(k):=\operatorname{ind}_{G\left(\mathbb{F}_{q}\right)}^{G}(k)$. While this $G$-module is infinite dimensional, it provides a potential way to relate extensions over $G\left(\mathbb{F}_{q}\right)$ with extensions over $G$. Indeed, by Generalized Frobenius Reciprocity and the fact that $G / G\left(\mathbb{F}_{q}\right)$ is affine, we have the following key observation.

Proposition . Let $M, N$ be rational $G$-modules. Then, for all $i \geq 0$,

$$
\operatorname{Ext}_{G\left(\mathbb{F}_{q}\right)}^{i}(M, N) \cong \operatorname{Ext}_{G}^{i}\left(M, N \otimes \mathcal{G}_{r}(k)\right) \text {. }
$$

2.4 Good Filtrations. To make the desired computations of cohomology groups, we will make use of Proposition 2.3 (with $M=k=N$ ). In addition, we will use a special filtration on $\mathcal{G}_{r}(k)$. Recall that a $G$-module $M$ has a good filtration if it admits a filtration with successive quotients of the form $H^{0}(\lambda), \lambda \in X(T)_{+}$[J1, II 4.16].

One may consider $k[G]$ as a $G \times G$-module via the left and right regular actions, respectively. A result due to Donkin and Koppinen [J1, II 4.20] now says that $k[G]$ as a $G \times G$-module admits a good filtration with factors of the form $H^{0}(\lambda) \otimes H^{0}\left(\lambda^{*}\right)$, each $\lambda \in X(T)_{+}$appearing exactly once. Here $\left(g_{1}, g_{2}\right) \in G \times G$ acts via $g_{1} \otimes g_{2}$ on each factor. If one takes the diagonal embedding of $G$ into $G \times G$ one can use this fact to show that $k[G]$ admits a good filtration under the adjoint action of $G$.

For our purposes, we modify this slightly by using a partial Frobenius twist. Consider the composite

$$
\phi: G \stackrel{\text { diag }}{\rightarrow} G \times G \stackrel{1 \times F^{r}}{\rightarrow} G \times G .
$$


That is, when we take the diagonal embedding, we apply the Frobenius morphism $r$-times to the second factor giving $\phi(g)=\left(g, F^{r}(g)\right)$. Let $G \times G$ act on $k[G]$ via the left and right regular representations as above, and then restrict this to a module over $G$ via $\phi$. Denote the resulting $G$-module by $k[G]^{\vee}$. The next proposition investigates filtrations on $k[G]^{\vee}$.

Proposition . As $G$-modules $\mathcal{G}_{r}(k) \cong k[G]^{\vee}$. Moreover, $\mathcal{G}_{r}(k)$ has a filtration with factors of the form $H^{0}(\lambda) \otimes H^{0}\left(\lambda^{*}\right)^{(r)}$ with multiplicity one for each $\lambda \in X(T)_{+}$.

Proof. Let $f \in k[G]$ and $g, x \in G$. If we denote the action of $G$ on $k[G]^{\vee}$ by $\star$ then

$$
(g \star f)(x)=f\left(g^{-1} x F^{r}(g)\right) .
$$

Next we define a Lang map $L: G \rightarrow G$ via $L(g)=g F^{r}\left(g^{-1}\right)$. By setting $L^{*}(f)=f \circ L$ one obtains a bijection ([Hum2, 1.4])

$$
L^{*}: k[G] \rightarrow \mathcal{G}_{r}(k)=\operatorname{ind}_{G\left(\mathbb{F}_{q}\right)}^{G}(k)=\left\{f \in k[G] \mid f(g h)=f(g) \text { for all } g \in G, h \in G\left(\mathbb{F}_{q}\right)\right\} .
$$

Observe that

$$
L^{*}(g \star f)(x)=(g \star f)(L(x))=f\left(g^{-1} L(x) F^{r}(g)\right)
$$

while

$$
\begin{aligned}
\left(g\left(L^{*}(f)\right)\right)(x) & =L^{*}(f)\left(g^{-1} x\right)=f\left(L\left(g^{-1} x\right)\right)=f\left(g^{-1} x F^{r}\left(x^{-1} g\right)\right) \\
& =f\left(g^{-1} x F^{r}\left(x^{-1}\right) F r^{r}(g)\right)=f\left(g^{-1} L(x) F^{r}(g)\right) .
\end{aligned}
$$

Hence, $L^{*}$ is a $G$-equivariant bijective map from $k[G]^{\vee}$ onto $\mathcal{G}_{r}(k)$.

Since $k[G]$, viewed as a $G \times G$-module, has a good filtration with factors $H^{0}(\lambda) \otimes H^{0}\left(\lambda^{*}\right)$ and $\phi(g)$ acts on each factor via $g \otimes F^{r}(g)$, it follows that $k[G]^{\vee}$ has a filtration with factors of the form $H^{0}(\lambda) \otimes H^{0}\left(\lambda^{*}\right)^{(r)}$ and multiplicity one for each $\lambda \in X(T)_{+}$, as claimed.

2.5 Given weights $\lambda, \mu \in X(T)$, recall that we say $\mu<\lambda$ if and only if $\lambda-\mu=\sum_{\alpha \in \Delta} c_{\alpha} \alpha$ for integers $c_{\alpha} \geq 0$. That is, $\lambda-\mu$ must lie in the positive root lattice. In addition we say two weights $\lambda$ and $\mu$ are linked if there exists an element $w$ of the affine Weyl group such that $\mu=w \cdot \lambda$. Note that two weights $\left(\lambda_{1}, \lambda_{2}\right),\left(\mu_{1}, \mu_{2}\right) \in X(T \times T)$ are linked for the algebraic group $G \times G$ if and only if the components are linked for $G$.

For each dominant weight $\lambda$ we define two finite saturated sets, namely

$$
\pi_{<\lambda}=\left\{\mu \in X(T)_{+} \mid \mu<\lambda\right\} \text { and } \pi_{\leq \lambda}=\left\{\mu \in X(T)_{+} \mid \mu \leq \lambda\right\} .
$$

According to [J1, II A.15] the $G \times G$-module $k[G]$ has two submodules $M_{<\lambda}$ and $M_{\leq \lambda}$, both admitting good filtrations with factors $H^{0}(\nu) \otimes H^{0}\left(\nu^{*}\right)$ where each $\nu \in \pi_{<\lambda}\left(\pi_{\leq \lambda}\right.$, respectively) appears exactly once. By $S_{<\lambda}$ and $S_{\leq \lambda}$ we denote the $G \times G$-summands of $M_{<\lambda}$ and $M_{\leq \lambda}$, respectively, whose $G \times G$-composition factors have highest weights contained in the same $G \times G$-linkage class as $\left(\lambda, \lambda^{*}\right)$. Similarly we define the quotients $Q_{\nless \lambda}=k[G] / S_{<\lambda}$ and $Q_{\not \lambda}=k[G] / S_{\leq \lambda}$.

The group $G$ acts on these modules via the embedding $\phi$. From Proposition 2.4 and [J1, II 4.17] one obtains the following result.

Theorem . For each $\lambda \in X(T)_{+}$, there exist short exact sequences of $G$-modules

$$
0 \rightarrow S_{<\lambda} \rightarrow \mathcal{G}_{r}(k) \rightarrow Q_{\nless \lambda} \rightarrow 0
$$


and

with the following properties:

$$
0 \rightarrow S_{\leq \lambda} \rightarrow \mathcal{G}_{r}(k) \rightarrow Q_{\not \lambda \lambda} \rightarrow 0
$$

(a) $S_{<\lambda}\left(S_{\leq \lambda}\right)$ has a filtration with factors of the form $H^{0}(\nu) \otimes H^{0}\left(\nu^{*}\right)^{(r)}$ where $\nu<\lambda$ $(\nu \leq \lambda)$ and $\nu$ is linked to $\lambda$.

(b) $Q_{\nless \lambda}\left(Q_{\nless \lambda}\right)$ has a filtration with factors of the form $H^{0}(\nu) \otimes H^{0}\left(\nu^{*}\right)^{(r)}$ where $\nu \nless \lambda$ $(\nu \nless \lambda)$ or $\nu$ is not linked to $\lambda$.

(c) The multiplicity in all cases is one.

2.6 Upper Bounds for $\operatorname{Ext}_{G\left(\mathbb{F}_{q}\right)}^{i}$ and Vanishing Criteria. The next theorem and its corollaries illustrate the usefulness of the existence of the filtrations in Proposition 2.4.

Theorem . Let $M, N$ be rational $G$-modules and $i \geq 0$. Then

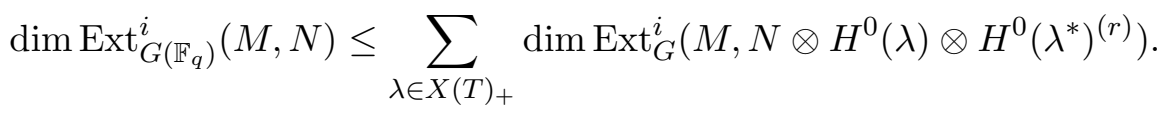

Proof. The result follows immediately from Proposition 2.3, Proposition 2.4 and the long exact sequence in cohomology associated to a short exact sequence.

One obtains the following vanishing criterion.

Corollary (A). Let $M, N$ be rational $G$-modules and $i \geq 0$. If $\operatorname{Ext}_{G}^{i}\left(M, N \otimes H^{0}(\lambda) \otimes\right.$ $\left.H^{0}\left(\lambda^{*}\right)^{(r)}\right)=0$ for all $\lambda \in X(T)_{+}$, then $\operatorname{Ext}_{G\left(\mathbb{F}_{q}\right)}^{i}(M, N)=0$.

In particular, in the special case of $M=k=N$, we get the following criterion for a vanishing range in cohomology. We will see later how to identify such an $m$ for a given $G$.

Corollary (B). Let $m$ be the least positive integer such that there exists $\lambda \in X(T)_{+}$with $\mathrm{H}^{m}\left(G, H^{0}(\lambda) \otimes H^{0}\left(\lambda^{*}\right)^{(r)}\right) \neq 0$. Then $\mathrm{H}^{i}\left(G\left(\mathbb{F}_{q}\right), k\right) \cong \mathrm{H}^{i}\left(G, \mathcal{G}_{r}(k)\right)=0$ for $0<i<m$.

2.7 Non-vanishing. While the identification of an $m$ satisfying Corollary 2.6(B) gives a vanishing range, it does not a priori follow that $\mathrm{H}^{m}\left(G\left(\mathbb{F}_{q}\right), k\right) \neq 0$. In this section, we develop some conditions under which this conclusion could be made, as well as conditions under which one might be able to precisely identify the cohomology group. As in Corollary 2.6(A), we have the following.

Proposition (A). Let $\lambda \in X(T)_{+}$and $M \in\left\{S_{<\lambda}, S_{\leq \lambda}, Q_{\nless \lambda}, Q_{\nless}\right\}$. Suppose that

$$
\mathrm{H}^{i}\left(G, H^{0}(\nu) \otimes H^{0}\left(\nu^{*}\right)^{(r)}\right)=0
$$

for all $\nu \in X(T)_{+}$which appear in the filtration for $M$. Then $\mathrm{H}^{i}(G, M)=0$.

The next proposition reduces the problem of showing the non-vanishing of $\mathrm{H}^{m}\left(G, \mathcal{G}_{r}(k)\right)$ (and hence of $\mathrm{H}^{m}\left(G\left(\mathbb{F}_{q}\right), k\right)$ ) to showing non-vanishing for a submodule of $\mathcal{G}_{r}(k)$.

Proposition (B). Let $m$ be a positive integer. For any $\lambda \in X(T)_{+}$,

(i) if $\mathrm{H}^{m+1}\left(G, S_{<\lambda}\right)=0$, then the map $\mathrm{H}^{m}\left(G, \mathcal{G}_{r}(k)\right) \rightarrow \mathrm{H}^{m}\left(G, Q_{\nless \lambda}\right)$ is surjective;

(ii) if $\mathrm{H}^{m}\left(G, S_{<\lambda}\right)=0$, then the map $\mathrm{H}^{m}\left(G, \mathcal{G}_{r}(k)\right) \rightarrow \mathrm{H}^{m}\left(G, Q_{\nless \lambda}\right)$ is injective; 
(iii) if the conditions in (i) and (ii) hold, then $\mathrm{H}^{m}\left(G, \mathcal{G}_{r}(k)\right) \cong \mathrm{H}^{m}\left(G, Q_{\nless \lambda}\right)$.

Proof. Consider the short exact sequence

$$
0 \rightarrow S_{<\lambda} \rightarrow \mathcal{G}_{r}(k) \rightarrow Q_{\nless \lambda} \rightarrow 0
$$

and the associated long exact sequence in cohomology

$$
\cdots \rightarrow \mathrm{H}^{m}\left(G, S_{<\lambda}\right) \rightarrow \mathrm{H}^{m}\left(G, \mathcal{G}_{r}(k)\right) \rightarrow \mathrm{H}^{m}\left(G, Q_{\nless \lambda}\right) \rightarrow \mathrm{H}^{m+1}\left(G, S_{<\lambda}\right) \rightarrow \cdots .
$$

The claims in (i) and (ii) follow immediately from this exact sequence.

Remark . In order to show the non-vanishing of $\mathrm{H}^{m}\left(G\left(\mathbb{F}_{q}\right), k\right)$, condition (i) is the crucial condition. Whereas condition (ii) potentially allows us to identify $\mathrm{H}^{m}\left(G\left(\mathbb{F}_{q}\right), k\right)$ precisely. Note that condition (ii) is immediately satisfied by any weight $\lambda$ which is minimal with respect to the above standard ordering such that $\mathrm{H}^{m}\left(G, H^{0}(\lambda) \otimes H^{0}\left(\lambda^{*}\right)^{(r)}\right) \neq 0$.

Having reduced the problem to $\mathrm{H}^{i}\left(G, Q_{\nless \lambda}\right)$, we make some similar homological observations about this group.

Proposition (C). Let $m$ be the least positive integer such that there exists $\nu \in X(T)_{+}$ with $\mathrm{H}^{m}\left(G, H^{0}(\nu) \otimes H^{0}\left(\nu^{*}\right)^{(r)}\right) \neq 0$. For any $\lambda \in X(T)_{+}$,

(i) the map $\mathrm{H}^{m}\left(G, H^{0}(\lambda) \otimes H^{0}\left(\lambda^{*}\right)^{(r)}\right) \rightarrow \mathrm{H}^{m}\left(G, Q_{\nless \lambda}\right)$ is injective;

(ii) if $\mathrm{H}^{m}\left(G, Q_{\nless \lambda}\right)=0$, then $\mathrm{H}^{m}\left(G, H^{0}(\lambda) \otimes H^{0}\left(\lambda^{*}\right)^{(r)}\right) \cong \mathrm{H}^{m}\left(G, Q_{\nless \lambda}\right)$.

Proof. Consider the short exact sequence

$$
0 \rightarrow H^{0}(\lambda) \otimes H^{0}\left(\lambda^{*}\right)^{(r)} \rightarrow Q_{\nless \lambda} \rightarrow Q_{\not \lambda \lambda} \rightarrow 0
$$

and the associated LES

$$
\cdots \rightarrow \mathrm{H}^{m-1}\left(G, Q_{\Varangle \lambda}\right) \rightarrow \mathrm{H}^{m}\left(G, H^{0}(\lambda) \otimes H^{0}\left(\lambda^{*}\right)^{(r)}\right) \rightarrow \mathrm{H}^{m}\left(G, Q_{\nless \lambda}\right) \rightarrow \mathrm{H}^{m}\left(G, Q_{\Varangle \lambda}\right) \rightarrow \cdots .
$$

If $m>1$, the claims follow immediately since the first term is zero by minimality of $m$.

Suppose that $m=1$. Suppose first that $\lambda$ is linked to the zero weight. Since we certainly cannot have $0>\lambda$, by Theorem $2.5(\mathrm{~b}), \mathrm{H}^{0}\left(G, Q_{\Varangle \lambda}\right)=\operatorname{Hom}_{G}\left(k, Q_{\nless \lambda}\right)=0$, and the argument follows as above.

Suppose now that $\lambda$ is not linked to the zero weight. The module $Q_{\nless \lambda}$ may be decomposed as a direct sum $M_{1} \oplus M_{2}$ where $M_{1}$ has a filtration with factors of the form $H^{0}(\nu) \otimes H^{0}\left(\nu^{*}\right)^{(1)}$ with $\nu$ linked to $\lambda$ and $M_{2}$ has such a filtration with $\nu$ not linked to $\lambda$. Then $\mathrm{H}^{1}\left(G, Q_{\nless \lambda}\right)=$ $\mathrm{H}^{1}\left(G, M_{1}\right) \oplus \mathrm{H}^{1}\left(G, M_{2}\right)$. Consider the short exact sequence

$$
0 \rightarrow H^{0}(\lambda) \otimes H^{0}\left(\lambda^{*}\right)^{(1)} \rightarrow M_{1} \rightarrow M_{1}^{\prime} \rightarrow 0,
$$

which defines a module $M_{1}^{\prime}$ with a corresponding filtration. Observe that $Q_{\not \lambda} \cong M_{1}^{\prime} \oplus M_{2}$, and hence $\mathrm{H}^{1}\left(G, Q_{\nless \lambda}\right) \cong \mathrm{H}^{1}\left(G, M_{1}^{\prime}\right) \oplus \mathrm{H}^{1}\left(G, M_{2}\right)$. Since $\lambda$ is not linked to zero, $\mathrm{H}^{0}\left(G, M_{1}^{\prime}\right)=$ $\operatorname{Hom}_{G}\left(k, M_{1}^{\prime}\right)=0$. Arguing as above with the LES associated to (2.7.1) gives

$$
\mathrm{H}^{1}\left(G, H^{0}(\lambda) \otimes H^{0}\left(\lambda^{*}\right)^{(1)}\right) \hookrightarrow \mathrm{H}^{1}\left(G, M_{1}\right) \hookrightarrow \mathrm{H}^{1}\left(G, Q_{\nless \lambda}\right),
$$

and so part (i) holds. For part (ii), it follows from the additional assumption that $\mathrm{H}^{1}\left(G, M_{1}^{\prime}\right)=$ 0 and $\mathrm{H}^{1}\left(G, M_{2}\right)=0$. Again using the LES associated to (2.7.1), it follows that

$$
\mathrm{H}^{1}\left(G, H^{0}(\lambda) \otimes H^{0}\left(\lambda^{*}\right)^{(1)}\right) \cong \mathrm{H}^{1}\left(G, M_{1}\right)=\mathrm{H}^{1}\left(G, M_{1}\right) \oplus \mathrm{H}^{1}\left(G, M_{2}\right) \cong \mathrm{H}^{1}\left(G, Q_{\nless \lambda}\right)
$$


as claimed.

2.8 Combining the propositions in the preceding section, we can obtain a condition under which we have sharp vanishing bounds, and an explicit identification of $\mathrm{H}^{m}\left(G\left(\mathbb{F}_{q}\right), k\right)$ with a single $G$-cohomology group.

Theorem (A). Let $m$ be the least positive integer such that there exists $\nu \in X(T)_{+}$with $\mathrm{H}^{m}\left(G, H^{0}(\nu) \otimes H^{0}\left(\nu^{*}\right)^{(r)}\right) \neq 0$. Let $\lambda \in X(T)_{+}$be such that $\mathrm{H}^{m}\left(G, H^{0}(\lambda) \otimes H^{0}\left(\lambda^{*}\right)^{(r)}\right) \neq 0$. Suppose $\mathrm{H}^{m+1}\left(G, H^{0}(\nu) \otimes H^{0}\left(\nu^{*}\right)^{(r)}\right)=0$ for all $\nu<\lambda$ that are linked to $\lambda$. Then

(i) $\mathrm{H}^{i}\left(G\left(\mathbb{F}_{q}\right), k\right)=0$ for $0<i<m$;

(ii) $\mathrm{H}^{m}\left(G\left(\mathbb{F}_{q}\right), k\right) \neq 0$;

(iii) if, in addition, $\mathrm{H}^{m}\left(G, H^{0}(\nu) \otimes H^{0}\left(\nu^{*}\right)^{(r)}\right)=0$ for all $\nu \in X(T)_{+}$with $\nu \neq \lambda$, then $\mathrm{H}^{m}\left(G\left(\mathbb{F}_{q}\right), k\right) \cong \mathrm{H}^{m}\left(G, H^{0}(\lambda) \otimes H^{0}\left(\lambda^{*}\right)^{(r)}\right)$.

Proof. Part (i) follows from Corollary 2.6(B). For part (ii), note first that, by the hypothesis on $\lambda$ and Proposition $2.7(\mathrm{C})(\mathrm{i}), \mathrm{H}^{m}\left(G, Q_{\nless \lambda}\right) \neq 0$. On the other hand, by the hypothesis on weights less than $\lambda$ and Proposition $2.7(\mathrm{~A}), \mathrm{H}^{m+1}\left(G, S_{<\lambda}\right)=0$. Hence, by Proposition 2.7(B)(i), the map $\mathrm{H}^{m}\left(G, \mathcal{G}_{r}(k)\right) \rightarrow \mathrm{H}^{m}\left(G, Q_{\nless \lambda}\right)$ is surjective. Therefore, by Proposition $2.3, \mathrm{H}^{m}\left(G\left(\mathbb{F}_{q}\right), k\right) \cong \mathrm{H}^{m}\left(G, \mathcal{G}_{r}(k)\right) \neq 0$ as claimed.

For part (iii), by the added assumption and Proposition 2.7(A), we have $\mathrm{H}^{m}\left(G, S_{<\lambda}\right)=$ 0 and $\mathrm{H}^{m}\left(G, Q_{\nless \lambda}\right)=0$. By Proposition 2.7(B)(iii) and Proposition 2.7(C)(ii), we have $\mathrm{H}^{m}\left(G, \mathcal{G}_{r}(k)\right) \cong \mathrm{H}^{m}\left(G, Q_{\nless \lambda}\right) \cong \mathrm{H}^{m}\left(G, H^{0}(\lambda) \otimes H^{0}\left(\lambda^{*}\right)^{(r)}\right)$ as claimed.

From the filtration on $\mathcal{G}_{r}(k)$ in Proposition $2.4, \mathrm{H}^{i}\left(G\left(\mathbb{F}_{q}\right), k\right) \cong \mathrm{H}^{i}\left(G, \mathcal{G}_{r}(k)\right)$ can be decomposed as a direct sum over linkage classes of dominant weights. As such, using an analogous argument, a slightly weaker condition for non-vanishing can be obtained.

Theorem (B). For a fixed linkage class $\mathcal{L}$, let $m$ be the least positive integer such that there exists $\nu \in \mathcal{L}$ with $\mathrm{H}^{m}\left(G, H^{0}(\nu) \otimes H^{0}\left(\nu^{*}\right)^{(r)}\right) \neq 0$. Let $\lambda \in \mathcal{L}$ be such that $\mathrm{H}^{m}\left(G, H^{0}(\lambda) \otimes\right.$ $\left.H^{0}\left(\lambda^{*}\right)^{(r)}\right) \neq 0$. Suppose $\mathrm{H}^{m+1}\left(G, H^{0}(\nu) \otimes H^{0}\left(\nu^{*}\right)^{(r)}\right)=0$ for all $\nu<\lambda$ in $\mathcal{L}$. Then $\mathrm{H}^{m}\left(G\left(\mathbb{F}_{q}\right), k\right) \neq 0$.

\section{Properties of the Cohomology Groups}

In Section 2, it was shown that knowledge of cohomology groups of the form $\mathrm{H}^{i}\left(G, H^{0}(\nu) \otimes\right.$ $\left.H^{0}\left(\nu^{*}\right)^{(r)}\right)$ for $\nu \in X(T)_{+}$provides information on $\mathrm{H}^{i}\left(G\left(\mathbb{F}_{q}\right), k\right)$. In this section, we study these $G$-cohomology groups and collect a number of useful properties that will be used throughout the remainder of the paper.

3.1 Reducing to $G_{r}$-cohomology. We will make frequent use of the following identification of $G$-extensions with $G_{r}$-cohomology.

Lemma . Let $\nu_{1}, \nu_{2} \in X(T)_{+}$. Assume that $\mathrm{H}^{j}\left(G_{r}, H^{0}\left(\nu_{1}\right)\right)^{(-r)}$ admits a good filtration for all $j>0$. Then for all $j$

$\mathrm{H}^{j}\left(G, H^{0}\left(\nu_{1}\right) \otimes H^{0}\left(\nu_{2}^{*}\right)^{(r)}\right) \cong \operatorname{Ext}_{G}^{j}\left(V\left(\nu_{2}\right)^{(r)}, H^{0}\left(\nu_{1}\right)\right) \cong \operatorname{Hom}_{G}\left(V\left(\nu_{2}\right), \mathrm{H}^{j}\left(G_{r}, H^{0}\left(\nu_{1}\right)\right)^{(-r)}\right)$. 
Proof. The first isomorphism is immediate. For the crucial second isomorphism, consider the Lyndon-Hochschild-Serre spectral sequence

$$
E_{2}^{i, j}=\operatorname{Ext}_{G / G_{r}}^{i}\left(V\left(\nu_{2}\right)^{(r)}, \mathrm{H}^{j}\left(G_{r}, H^{0}\left(\nu_{1}\right)\right)\right) \Rightarrow \operatorname{Ext}_{G}^{i+j}\left(V\left(\nu_{2}\right)^{(r)}, H^{0}\left(\nu_{1}\right)\right) .
$$

We have

$$
\begin{aligned}
E_{2}^{i, j} & =\operatorname{Ext}_{G / G_{r}}^{i}\left(V\left(\nu_{2}\right)^{(r)}, \mathrm{H}^{j}\left(G_{r}, H^{0}\left(\nu_{1}\right)\right)\right) \\
& \cong \operatorname{Ext}_{G}^{i}\left(V\left(\nu_{2}\right), \mathrm{H}^{j}\left(G_{r}, H^{0}\left(\nu_{1}\right)\right)^{(-r)}\right) .
\end{aligned}
$$

For $\nu \in X(T)_{+}, i>0$, and $V$ a $G$-module which admits a good filtration, we have $\operatorname{Ext}_{G}^{i}(V(\nu), V)=0$ (cf. [J1, Prop. II 4.16]). By the hypothesis, we conclude that $E_{2}^{i, j}=0$ for all $i>0$ and the spectral sequence collapses to a single vertical column. This implies that $E_{2}^{0, j} \cong \operatorname{Ext}_{G}^{j}\left(V\left(\nu_{2}\right)^{(r)}, H^{0}\left(\nu_{1}\right)\right)$ for all $j$.

The assumption that $\mathrm{H}^{j}\left(G_{r}, H^{0}(\nu)\right)^{(-r)}$ admits a good filtration is a long-standing conjecture of Donkin. For $p>h$ (the Coxeter number of the root system associated to $G$ ), this is known for $r=1$ by results of Andersen-Jantzen [AJ] and Kumar-Lauritzen-Thomsen KLT]. For arbitrary $r$, this is known only for all degrees in the case $G=S L_{2}$. When $r$ is arbitrary and $i=1$ ( $p$ arbitrary) or $i=2(p \geq 3)$, Bendel-Nakano-Pillen verified the assumption by direct computation [BNP4, BNP7]. Wright [W] has recently verified the $p=2$, $i=2$ case.

We will apply Lemma 3.1 at several points in the $r=1$ case for direct applications to $G\left(\mathbb{F}_{p}\right)$ as well as inductively for dealing with $G\left(\mathbb{F}_{q}\right)$. As such, we will generally assume for the remainder of the paper that $p>h$.

3.2 Dimensions for $r=1$. From Lemma 3.1, to obtain information about $\mathrm{H}^{i}\left(G, H^{0}(\nu) \otimes\right.$ $\left.H^{0}\left(\nu^{*}\right)^{(1)}\right)$ for $\nu \in X(T)_{+}$, it suffices to consider $\operatorname{Hom}_{G}\left(V(\nu), \mathrm{H}^{i}\left(G_{1}, H^{0}(\nu)^{(-1)}\right)\right.$. It is wellknown that, from block considerations, $\mathrm{H}^{i}\left(G_{1}, H^{0}(\nu)\right)=0$ unless $\nu=w \cdot 0+p \mu$ for $w \in W$ and $\mu \in X(T)$. For $p>h$, from [AJ] and [KLT], we have

$$
\mathrm{H}^{i}\left(G_{1}, H^{0}(\nu)\right)^{(-1)}= \begin{cases}\operatorname{ind}_{B}^{G}\left(S^{\frac{i-\ell(w)}{2}}\left(\mathfrak{u}^{*}\right) \otimes \mu\right) & \text { if } \nu=w \cdot 0+p \mu \\ 0 & \text { otherwise, }\end{cases}
$$

where $\mathfrak{u}=\operatorname{Lie}(U)$. Note also that, since $p>h$ and $\nu$ is dominant, $\mu$ must also be dominant.

For a weight $\nu$ and $n \geq 0$, let $P_{n}(\nu)$ denote the dimension of the $\nu$-weight space of $S^{n}\left(\mathfrak{u}^{*}\right)$. Equivalently, for $n>0, P_{n}(\nu)$ denotes the number of times that $\nu$ can be expressed as a sum of exactly $n$ positive roots, while $P_{0}(0)=1$. The function $P_{n}$ is often referred to as Kostant's Partition Function. By using [AJ, 3.8], [KLT, Thm 2], Lemma 3.1, and (3.2.1), we can give an explicit formula for the dimension of $\mathrm{H}^{i}\left(G, H^{0}(\lambda) \otimes H^{0}\left(\lambda^{*}\right)^{(1)}\right)$. Namely,

Proposition . Assume $p>h$. Let $\lambda=p \mu+w \cdot 0 \in X(T)_{+}$. Then

$$
\operatorname{dim} H^{i}\left(G, H^{0}(\lambda) \otimes H^{0}\left(\lambda^{*}\right)^{(1)}\right)=\sum_{u \in W}(-1)^{\ell(u)} P_{\frac{i-\ell(w)}{2}}(u \cdot \lambda-\mu) .
$$

3.3 From Proposition 2.3 and Proposition 2.4, we can now deduce the following upper bound on the dimensions of the cohomology groups $\mathrm{H}^{i}\left(G\left(\mathbb{F}_{q}\right), k\right)$. 
Theorem . Assume $p>h$.

$$
\operatorname{dim} H^{i}\left(G\left(\mathbb{F}_{p}\right), k\right) \leq \sum_{\{w \in W \mid \ell(w) \equiv i \bmod 2\}} \sum_{\mu \in X(T)_{+}} \sum_{u \in W}(-1)^{\ell(u)} P_{\frac{i-\ell(w)}{2}}(u \cdot(p \mu+w \cdot 0)-\mu) .
$$

3.4 Degree Bounds. From our discussion in Section 2, to find vanishing ranges for $\mathrm{H}^{\bullet}\left(G\left(\mathbb{F}_{p}\right), k\right)$ (or $\mathrm{H}^{\bullet}\left(G\left(\mathbb{F}_{q}\right), k\right)$ more generally), a first step is to try to identify the least positive $i$ such that $\mathrm{H}^{i}\left(G, H^{0}(\lambda) \otimes H^{0}\left(\lambda^{*}\right)^{(1)}\right)$ is non-zero.

Assume that $p>h$ and $\lambda \in X(T)_{+}$with $\mathrm{H}^{i}\left(G, H^{0}(\lambda) \otimes H^{0}\left(\lambda^{*}\right)^{(1)}\right) \neq 0$ for some $i>0$. From Lemma 3.1 and the discussion in Section 3.2, we know that $\lambda=p \mu+w \cdot 0$ for $w \in W$ and $\mu \in X(T)_{+}$. Observe that if $\mu=0$, in order for $\lambda$ to be dominant, we must have $\lambda=0$. But, $\mathrm{H}^{i}(G, k)=0$ for $i>0$. Since we are interested in cohomology in non-zero degrees, we may safely assume that $\lambda, \mu \neq 0$. Corollary 3.5 below gives a relationship between $i$ and the weight $\lambda$. We first derive a more general relationship that will be useful in inductive arguments.

Proposition . Assume that $p>h$. Let $\gamma_{1}, \gamma_{2} \in X(T)_{+}$, both non-zero, such that $\gamma_{j}=$ $p \delta_{j}+w_{j} \cdot 0$ with $\delta_{j} \in X(T)_{+}$and $w_{j} \in W$ for $j=1,2$. Assume $\operatorname{Ext}_{G}^{i}\left(V\left(\gamma_{2}\right)^{(1)}, H^{0}\left(\gamma_{1}\right)\right) \neq 0$.

(a) Let $\sigma \in \Phi^{+}$. If $\Phi$ is of type $G_{2}$, assume further that $\sigma$ is a long root. Then $p\left\langle\delta_{2}, \sigma^{\vee}\right\rangle-\left\langle\delta_{1}, \sigma^{\vee}\right\rangle+\ell\left(w_{1}\right)+\left\langle w_{2} \cdot 0, \sigma^{\vee}\right\rangle \leq i$.

(b) Let $\tilde{\alpha}$ denote the longest root in $\Phi^{+}$. Then $p\left\langle\delta_{2}, \tilde{\alpha}^{\vee}\right\rangle-\left\langle\delta_{1}, \tilde{\alpha}^{\vee}\right\rangle+\ell\left(w_{1}\right)-\ell\left(w_{2}\right)-1 \leq i$. Equality requires that $\gamma_{2}-\delta_{1}=\left(\left(i-l\left(w_{1}\right)\right) / 2\right) \tilde{\alpha}$ and $\left\langle-w_{2} \cdot 0, \tilde{\alpha}^{\vee}\right\rangle=\ell\left(w_{2}\right)+1$.

Proof. By Lemma 3.1, (3.2.1), and Frobenius reciprocity, we have

$$
\begin{aligned}
\operatorname{Ext}_{G}^{i}\left(V\left(\gamma_{2}\right)^{(1)}, H^{0}\left(\gamma_{1}\right)\right) & \cong \operatorname{Hom}_{G}\left(V\left(\gamma_{2}\right), H^{i}\left(G_{1}, H^{0}\left(\gamma_{1}\right)\right)^{(-1)}\right) \\
& \cong \operatorname{Hom}_{G}\left(V\left(\gamma_{2}\right), \operatorname{ind}_{B}^{G}\left(S^{\frac{i-\ell\left(w_{1}\right)}{2}}\left(\mathfrak{u}^{*}\right) \otimes \delta_{1}\right)\right) \\
& \cong \operatorname{Hom}_{B}\left(V\left(\gamma_{2}\right), S^{\frac{i-\ell\left(w_{1}\right)}{2}}\left(\mathfrak{u}^{*}\right) \otimes \delta_{1}\right) .
\end{aligned}
$$

Since this is non-zero, $\gamma_{2}=p \delta_{2}+w_{2} \cdot 0$ must be a weight of $S^{\frac{i-\ell\left(w_{1}\right)}{2}}\left(\mathfrak{u}^{*}\right) \otimes \delta_{1}$. In other words, $\gamma_{2}-\delta_{1}=p \delta_{2}-\delta_{1}+w_{2} \cdot 0$ must be a weight of $S^{\frac{i-\ell\left(w_{1}\right)}{2}}\left(\mathfrak{u}^{*}\right)$.

The vector space $\mathfrak{u}^{*}$ has a basis of root vectors corresponding to positive roots. So a homogeneous weight of $S^{j}\left(\mathfrak{u}^{*}\right)$ is a sum of $j$ not necessarily distinct positive roots. Therefore, $\gamma_{2}-\delta_{1}$ must be expressible as a sum of $\frac{i-\ell\left(w_{1}\right)}{2}$ positive roots. For any positive roots $\sigma_{1}, \sigma_{2}$ (with $\sigma_{2}$ being long if $\Phi$ is of type $G_{2}$ ), we have $\left\langle\sigma_{1}, \sigma_{2}^{\vee}\right\rangle \leq 2$. Hence, for $\sigma \in \Phi^{+}$, we have

$$
\left\langle\gamma_{2}-\delta_{1}, \sigma^{\vee}\right\rangle \leq \frac{i-\ell\left(w_{1}\right)}{2} * 2=i-\ell\left(w_{1}\right) .
$$

Substituting $\gamma_{2}-\delta_{1}=p \delta_{2}-\delta_{1}+w_{2} \cdot 0$ gives

$$
p\left\langle\delta_{2}, \sigma^{\vee}\right\rangle-\left\langle\delta_{1}, \sigma^{\vee}\right\rangle+\left\langle w_{2} \cdot 0, \sigma^{\vee}\right\rangle \leq i-\ell\left(w_{1}\right) .
$$

Part (a) immediately follows.

For part (b), Note that for $\sigma=\tilde{\alpha}$ equality in Equation (3.4.1) can only hold if $\gamma_{2}-\delta_{1}=$ $\left(\left(i-l\left(w_{1}\right)\right) / 2\right) \tilde{\alpha}$. This follows from Observation 2.2(A). In addition, by Observation 2.2(B), 
ON THE VANISHING RANGES FOR THE COHOMOLOGY OF FINITE GROUPS OF LIE TYPE

$-w_{2} \cdot 0$ can be expressed uniquely as a sum of precisely $\ell\left(w_{2}\right)$ distinct positive roots. Since at most one of those roots can be $\tilde{\alpha}$, and $\left\langle\tilde{\alpha}, \tilde{\alpha}^{\vee}\right\rangle=2$, by Observation 2.2(A), we have

$$
\left\langle-w_{2} \cdot 0, \tilde{\alpha}^{\vee}\right\rangle \leq\left(\ell\left(w_{2}\right)-1\right) * 1+2=\ell\left(w_{2}\right)+1
$$

which gives part (b).

3.5 As a special case of Proposition 3.4 we have the following result.

Corollary . Assume that $p>h$. Let $\lambda=p \mu+w \cdot 0$ be a non-zero dominant weight with $0 \neq \mu \in X(T)_{+}$and $w \in W$. Assume $\mathrm{H}^{i}\left(G, H^{0}(\lambda) \otimes H^{0}\left(\lambda^{*}\right)^{(1)}\right) \neq 0$.

(a) Let $\sigma \in \Phi^{+}$. If $\Phi$ is of type $G_{2}$, assume further that $\sigma$ is a long root. Then $(p-1)\left\langle\mu, \sigma^{\vee}\right\rangle+\ell(w)+\left\langle w \cdot 0, \sigma^{\vee}\right\rangle \leq i$.

(b) Let $\tilde{\alpha}$ denote the longest root in $\Phi^{+}$. Then $(p-1)\left\langle\mu, \tilde{\alpha}^{\vee}\right\rangle-1 \leq i$. Equality requires that $\lambda-\mu=((i-l(w)) / 2) \tilde{\alpha}$ and $\left\langle-w \cdot 0, \tilde{\alpha}^{\vee}\right\rangle=\ell(w)+1$.

Proof. Parts (a) and (b) follow immediately from Propositon 3.4 by taking $\gamma_{1}=\lambda=\gamma_{2}$.

In the corollary, since $\mu$ is a non-zero dominant weight, $\left\langle\mu, \tilde{\alpha}^{\vee}\right\rangle \geq 1$. Hence, we immediately have that $i \geq p-2$. It follows from Corollary 2.6(B) that $\mathrm{H}^{i}\left(G\left(\mathbb{F}_{p}\right), k\right)=0$ for $0<i<p-2$. This will follow as a special case of a more general result in the next section.

\section{A Minimal Vanishing Range}

4.1 In this section, we use the preceding techniques to determine a general vanishing range for $\mathrm{H}^{i}\left(G\left(\mathbb{F}_{q}\right), k\right)$ for $p>h$. We begin with some further extension properties that will be used in the proof.

Lemma . Assume that $p>h$ and $r>1$. Let $\lambda, \mu \in X(T)_{+}$, both non-zero, and $i \geq 0$. If $\operatorname{Ext}_{G}^{i}\left(V(\lambda)^{(r)}, H^{0}(\mu)\right) \neq 0$, then there exists a non-zero weight $\gamma \in X(T)_{+}$and nonnegative integers $k, l$ such that

(a) $i=k+l$,

(b) $\operatorname{Ext}_{G}^{k}\left(V(\lambda)^{(r-1)}, H^{0}(\gamma)\right) \neq 0$,

(c) $\operatorname{Ext}_{G}^{l}\left(V(\gamma)^{(1)}, H^{0}(\mu)\right) \neq 0$, and

(d) $\gamma=p \delta+w \cdot 0$ for some $w \in W$ and non-zero $\delta \in X(T)_{+}$.

Proof. Consider the Lyndon-Hochschild-Serre spectral sequence

$$
E_{2}^{k, l}=\operatorname{Ext}_{G / G_{1}}^{k}\left(V(\lambda)^{(r)}, \mathrm{H}^{l}\left(G_{1}, H^{0}(\mu)\right)\right) \Rightarrow \operatorname{Ext}_{G}^{k+l}\left(V(\lambda)^{(r)}, H^{0}(\mu)\right) .
$$

The assumptions imply that there exist nonnegative integers $k, l$ with $k+l=i$ and $\operatorname{Ext}_{G / G_{1}}^{k}\left(V(\lambda)^{(r)}, \mathrm{H}^{l}\left(G_{1}, H^{0}(\mu)\right)\right) \neq 0$. The $G$-module $\mathrm{H}^{l}\left(G_{1}, H^{0}(\mu)\right)^{(-1)}$ has a good filtration. Therefore, there exists a dominant weight $\gamma$ with

$$
\operatorname{Hom}_{G / G_{1}}\left(V(\gamma)^{(1)}, \mathrm{H}^{l}\left(G_{1}, H^{0}(\mu)\right)\right) \cong \operatorname{Hom}_{G}\left(V(\gamma), \mathrm{H}^{l}\left(G_{1}, H^{0}(\mu)\right)^{(-1)}\right) \neq 0
$$

and

$$
\operatorname{Ext}_{G}^{k}\left(V(\lambda)^{(r-1)}, H^{0}(\gamma)\right) \cong \operatorname{Ext}_{G / G_{1}}^{k}\left(V(\lambda)^{(r)}, H^{0}(\gamma)^{(1)}\right) \neq 0
$$


Now (4.1.2) implies that $\gamma$ is of the form $\gamma=p \delta+w \cdot 0$ with $w \in W$ and $\delta \in X(T)_{+}$. By Lemma 3.1 and (4.1.1), $\operatorname{Ext}_{G}^{l}\left(V(\gamma)^{(1)}, H^{0}(\mu)\right) \cong \operatorname{Hom}_{G}\left(V(\gamma), \mathrm{H}^{l}\left(G_{1}, H^{0}(\mu)\right)^{(-1)}\right) \neq 0$. This forces $\delta \neq 0$. Note that the assumptions also force $\mu=p \delta^{\prime}+w^{\prime} \cdot 0$ for some $w^{\prime} \in W$ and nonzero $\delta^{\prime} \in X(T)_{+}$.

4.2 Applying the lemma repeatedly immediately implies the following proposition.

Proposition . Assume that $p>h$. Let $\lambda, \mu \in X(T)_{+}$, both non-zero, and $i \geq 0$. If $\operatorname{Ext}_{G}^{i}\left(V(\lambda)^{(r)}, H^{0}(\mu)\right) \neq 0$, then there exists a sequence of non-zero weights $\mu=\gamma_{0}, \gamma_{1}, \ldots, \gamma_{r-1}, \gamma_{r}=\lambda \in X(T)_{+}$and nonnegative integers $l_{1}, l_{2}, \ldots, l_{r}$ such that

(a) $i=\sum_{j=1}^{r} l_{j}$

(b) $\operatorname{Ext}_{G}^{l_{j}}\left(V\left(\gamma_{j}\right)^{(1)}, H^{0}\left(\gamma_{j-1}\right)\right) \neq 0$, for $1 \leq j \leq r$ and

(c) $\gamma_{j}=p \delta_{j}+u_{j} \cdot 0$ with $u_{j} \in W$ and nonzero $\delta_{j} \in X(T)_{+}$, for $1 \leq j \leq r-1$.

4.3 The next step in our analysis is to obtain tighter control over a lower bound on $i$ as in Proposition 4.2 in the case that $\lambda=\mu$.

Proposition . Assume that $p>h$. Let $0 \neq \lambda \in X(T)_{+}$and $i \geq 0$. If $\mathrm{H}^{i}\left(G, H^{0}(\lambda) \otimes\right.$ $\left.H^{0}\left(\lambda^{*}\right)^{(r)}\right) \neq 0$, then there exists a sequence of non-zero weights $\lambda=\gamma_{0}, \gamma_{1}, \ldots, \gamma_{r-1}, \gamma_{r}=$ $\lambda \in X(T)_{+}$such that $\gamma_{j}=p \delta_{j}+u_{j} \cdot 0$ for some $u_{j} \in W$ and nonzero $\delta_{j} \in X(T)_{+}$. Furthermore,

$$
i \geq\left(\sum_{j=1}^{r}(p-1)\left\langle\delta_{j}, \tilde{\alpha}^{\vee}\right\rangle\right)-r .
$$

Equality requires that $p \delta_{j}-\delta_{j-1}+u_{j} \cdot 0=\left(\left(l_{j}-l\left(u_{j-1}\right)\right) / 2\right) \tilde{\alpha}$ and that $\left\langle-u_{j} \cdot 0, \tilde{\alpha}^{\vee}\right\rangle=\ell\left(u_{j}\right)+1$ for all $1 \leq j \leq r$, where $l_{j}$ is as in Proposition 4.2.

Proof. The first part is simply a partial restatement of Proposition 4.2 with $\lambda=\mu$. Specifically, there exists a sequence of non-zero dominant weights $\mathrm{v} \lambda=\gamma_{0}, \gamma_{1}, \ldots, \gamma_{r-1}, \gamma_{r}=\lambda$ with $\gamma_{j}=p \delta_{j}+u_{j} \cdot 0$ and corresponding nonnegative integers $l_{j}$ with $i=\sum_{j=1}^{r} l_{j}$ and $\operatorname{Ext}_{G}^{l_{j}}\left(V\left(\gamma_{j}\right)^{(1)}, H^{0}\left(\gamma_{j-1}\right)\right) \neq 0$.

For (4.3.1), we use Proposition 3.4(b) to obtain the inequalities

$$
p\left\langle\delta_{j}, \tilde{\alpha}^{\vee}\right\rangle-\left\langle\delta_{j-1}, \tilde{\alpha}^{\vee}\right\rangle+\ell\left(u_{j-1}\right)-\ell\left(u_{j}\right)-1 \leq l_{j} \text { for } 1 \leq j \leq r,
$$

with equality only if $p \delta_{j}-\delta_{j-1}+u_{j} \cdot 0=\left(\left(l_{j}-l\left(u_{j-1}\right)\right) / 2\right) \tilde{\alpha}$ and $\left\langle-u_{j} \cdot 0, \tilde{\alpha}^{\vee}\right\rangle=\ell\left(u_{j}\right)+1$ for all $1 \leq j \leq r$. Note that $\delta_{0}=\delta_{r}$ and $u_{0}=u_{r}$. Summing over $j$ yields

$$
\left(\sum_{j=1}^{r}(p-1)\left\langle\delta_{j}, \tilde{\alpha}^{\vee}\right\rangle\right)-r \leq \sum_{j=1}^{r} l_{j}=i .
$$

4.4 For $p>h$ we can now present general vanishing ranges which address (1.1.1).

Theorem . Assume that $p>h$. Then 
(a) $\mathrm{H}^{i}\left(G, H^{0}(\lambda) \otimes H^{0}\left(\lambda^{*}\right)^{(r)}\right)=0$ for $0<i<r(p-2)$ and $\lambda \in X(T)_{+}$;

(b) $\mathrm{H}^{i}\left(G\left(\mathbb{F}_{q}\right), k\right)=0$ for $0<i<r(p-2)$.

Proof. Part (a) implies part (b) via Corollary 2.6(B). Suppose that

$$
\mathrm{H}^{i}\left(G, H^{0}(\lambda) \otimes H^{0}\left(\lambda^{*}\right)^{(r)}\right) \neq 0
$$

for some $0<i$ and $\lambda \in X(T)_{+}$. Clearly $\lambda \neq 0$, so we may apply Proposition 4.3. Since $\gamma_{j} \neq 0,1 \leq\left\langle\delta_{j}, \tilde{\alpha}^{\vee}\right\rangle$. Proposition 4.3 then gives $i \geq r(p-1)-r=r(p-2)$ as claimed.

Observe that this vanishing range is generally larger than the one obtained in $[\mathrm{H}$. Precisely, the ranges obtained in $[\mathrm{H}]$ are of the form $0<i<m$ where $m$ depends on the root system. Except in certain type $A_{n}$ cases, $m \leq r(p-1) / 2$.

In the remainder of the paper, we further investigate this question to determine sharp bounds for root systems of type $C_{n}$ (for all $r$; see Theorem 5.4) and $A_{n}$ (for $r=1$ and generically for all $r$; see Theorems $6.13,6.14$ ). In type $C_{n}$, the above bounds are in fact sharp.

Remark . Note that the assumption $\operatorname{Ext}_{G}^{i}\left(V(\lambda)^{(r)}, H^{0}(\mu)\right) \neq 0$ in Proposition 4.2 can be replaced by $\operatorname{Ext}_{G / G_{1}}^{k}\left(V(\lambda)^{(r)}, \mathrm{H}^{l}\left(G_{1}, H^{0}(\mu)\right)\right) \neq 0$, where $k+l=i$. In that case one arrives at the same conclusions with $l_{1}=l$. Now the arguments used to prove Proposition 4.3 and Theorem 4.4 can be used to show that $\operatorname{Ext}_{G / G_{1}}^{k}\left(V(\lambda)^{(r)}, \mathrm{H}^{l}\left(G_{1}, H^{0}(\lambda)\right)=0\right.$ for all $k+l<r(p-2)$.

\section{Type $C_{n}, n \geq 1$}

Assume throughout this section that $\Phi$ is of type $C_{n}, n \geq 1$, and $p>h=2 n$.

5.1 Realization for $r=1$. We determine the least $i>0$ such that $\mathrm{H}^{i}\left(G, H^{0}(\lambda) \otimes\right.$ $\left.H^{0}\left(\lambda^{*}\right)^{(1)}\right) \neq 0$. From Theorem 4.4, we know that $i \geq p-2$. Let $\tilde{\alpha}=2 \omega_{1}$ denote the longest positive root. We next construct a weight $\lambda$ with $\mathrm{H}^{p-2}\left(G, H^{0}(\lambda) \otimes H^{0}\left(\lambda^{*}\right)^{(1)}\right) \neq 0$. Let $w=s_{\tilde{\alpha}}=s_{1} s_{2} \ldots s_{n-1} s_{n} s_{n-1} \ldots s_{2} s_{1} \in W$. Then $-w \cdot 0=n \tilde{\alpha}=2 n \omega_{1}$. Furthermore, when expressed as a sum of distinct positive roots, $-w \cdot 0$ consists of precisely all positive roots which contain an $\alpha_{1}$. Set $\lambda=p \omega_{1}+w \cdot 0=p \omega_{1}-2 n \omega_{1}=(p-2 n) \omega_{1}$. Then

$$
\lambda-\omega_{1}=(p-1-2 n) \omega_{1}=\left(\frac{p-1}{2}-n\right) \tilde{\alpha}
$$

is a highest weight of $S^{j}\left(\mathfrak{u}^{*}\right)$ where $j=\frac{p-1}{2}-n$.

We will apply Proposition 3.2 to compute $\operatorname{dim} \mathrm{H}^{p-2}\left(G, H^{0}(\lambda) \otimes H^{0}\left(\lambda^{*}\right)^{(1)}\right)$. Specifically, we will show that for $u \in W$

$$
P_{\frac{p-1}{2}-n}\left(u \cdot \lambda-\omega_{1}\right)=\left\{\begin{array}{l}
1 \text { if } u=1 \\
0 \text { else. }
\end{array}\right.
$$

We will work with the $\epsilon$-basis of $X(T)$. Rewrite $u \cdot \lambda-\omega_{1}=((p-2 n) u-1) \epsilon_{1}+u \cdot 0$ as $\sum_{i} c_{i} \epsilon_{i}$. In order for this expression to be a sum of positive roots, the coefficient $c_{1}$ has to be nonnegative. This forces $u\left(\epsilon_{1}\right)=\epsilon_{1}$. Then $u \cdot 0$ is of the form $-\sum_{i} d_{i} \alpha_{i}$ with $d_{1}=0$. This implies that $u \cdot \lambda-\omega_{1}=\left(\frac{p-1}{2}-n\right) \tilde{\alpha}-\sum_{i} d_{i} \alpha_{i}$. Such an expression contains $p-1-2 n$ 
copies of $\alpha_{1}$. Since $\tilde{\alpha}$ is the only positive root containing $2 \alpha_{1}$, the above expression can be written as a sum of $\frac{p-1}{2}-n$ positive roots if and only if $u=1$. From Proposition 3.2, one concludes that $\mathrm{H}^{p-2}\left(G, H^{0}(\lambda) \otimes H^{0}\left(\lambda^{*}\right)^{(1)}\right) \cong k$.

5.2 More Vanishing in Degree $p-2$. To get a precise vanishing range, we need to consider whether there are any other weights with non-zero cohomology in degree $p-2$. Let $\lambda=p \mu+w \cdot 0 \in X(T)_{+}$with $\mathrm{H}^{i}\left(G, H^{0}(\lambda) \otimes H^{0}\left(\lambda^{*}\right)^{(-1)}\right) \neq 0$ for some $i>0$. Consider the maximal short root $\alpha_{0}=\omega_{2}$. By Corollary 3.5(a) with $\sigma=\alpha_{0}$,

$$
i \geq(p-1)\left\langle\mu, \alpha_{0}^{\vee}\right\rangle+\ell(w)+\left\langle w \cdot 0, \alpha_{0}^{\vee}\right\rangle .
$$

There are three positive roots $\beta$ with $\left\langle\beta, \alpha_{0}^{\vee}\right\rangle=2$ (unless $n=1$, in which case there is only one and $\left.\alpha_{0}=2 \omega_{1}\right)$. Since $-w \cdot 0$ can be expressed uniquely as a sum of $\ell(w)$ distinct positive roots, we can say $\left\langle-w \cdot 0, \alpha_{0}^{\vee}\right\rangle \leq \ell(w)+3$. Hence, (5.2.1) can be rewritten as

$$
i \geq(p-1)\left\langle\mu, \alpha_{0}^{\vee}\right\rangle-3 \text {. }
$$

In Type $C_{n},\left\langle\omega_{1}, \alpha_{0}^{\vee}\right\rangle=1$. But, for $2 \leq j \leq n,\left\langle\omega_{j}, \alpha_{0}^{\vee}\right\rangle=2$. Hence, for $\mu \in X(T)_{+}$, if $\mu \neq 0, \omega_{1}$, then $\left\langle\mu, \alpha_{0}^{\vee}\right\rangle \geq 2$. If $\left\langle\mu, \alpha_{0}^{\vee}\right\rangle \geq 2$, then (5.2.2) becomes

$$
i \geq 2(p-1)-3=2 p-5>p-2
$$

since $p \geq 5(n \geq 2)$. Therefore, the only candidates for a non-zero cohomology group in degree $p-2$ are with $\lambda=p \omega_{1}+w \cdot 0$ for some $w \in W$. This makes sense because the weight constructed in Section 5.1 is of this form.

5.3 A Sharp Bound for $r=1$. Suppose $\lambda=p \omega_{1}+w \cdot 0 \in X(T)_{+}$and

$$
\mathrm{H}^{p-2}\left(G, H^{0}(\lambda) \otimes H^{0}\left(\lambda^{*}\right)^{(1)}\right) \neq 0 .
$$

Proposition 3.4 part (b) implies that $\lambda-\omega_{1}=(p-1) \omega_{1}+w \cdot 0=((p-1) / 2) \tilde{\alpha}+w \cdot 0=$ $((p-2-\ell(w)) / 2) \tilde{\alpha}$ and $\left\langle w \cdot 0, \tilde{\alpha}^{\vee}\right\rangle=-(\ell(w)+1)$. This forces $w \cdot 0=-((l(w)+1) / 2) \tilde{\alpha}$. The only possible choices for $w$ satisfying this last equation are $w=0$ and $w=s_{\tilde{\alpha}}$. Now $\left\langle w \cdot 0, \tilde{\alpha}^{\vee}\right\rangle=-(\ell(w)+1)$ forces $w=s_{\tilde{\alpha}}$ and $w \cdot 0=-n \tilde{\alpha}$. Hence, $\lambda=(p-2 n) \omega_{1}$, the weight given in Section 5.1. So the $\lambda$ exhibited there is the only dominant weight with $\mathrm{H}^{p-2}\left(G, H^{0}(\lambda) \otimes H^{0}\left(\lambda^{*}\right)^{(1)}\right) \neq 0$.

Note that $\lambda=(p-2 n) \omega_{1}$ is contained in the lowest alcove. There are no smaller dominant weights that are linked to $\lambda$ (so the condition in Theorem $2.8(\mathrm{~A})$ involving $\mathrm{H}^{m+1}$ is vacuous). Consequently, Theorem 2.8(A) and the above discussion now yields:

Theorem . Suppose $\Phi$ is of type $C_{n}$ with $p>2 n$. Then

(a) $\mathrm{H}^{i}\left(G\left(\mathbb{F}_{p}\right), k\right)=0$ for $0<i<p-2$;

(b) $\mathrm{H}^{p-2}\left(G\left(\mathbb{F}_{p}\right), k\right) \cong k$.

5.4 A Sharp Bound for all $r$. In this section we will address (1.1.1) and (1.1.2) in general for $\mathrm{H}^{\bullet}\left(G\left(\mathbb{F}_{q}\right), k\right)$ when $\Phi$ is of type $C_{n}$.

Lemma . Assume $p>2 n$. Let $\lambda=(p-2 n) \omega_{1}$. Then $\operatorname{Ext}_{G}^{r(p-2)}\left(V(\lambda)^{(r)}, H^{0}(\lambda)\right) \cong k$. 
Proof. We use induction on $r$. If $r=1$ the assertion follows from Section 5.1. Next we make use of the LHS spectral sequence

$$
E_{2}^{k, l}=\operatorname{Ext}_{G / G_{1}}^{k}\left(V(\lambda)^{(r)}, \mathrm{H}^{l}\left(G_{1}, H^{0}(\lambda)\right) \Rightarrow \operatorname{Ext}_{G}^{k+l}\left(V(\lambda)^{(r)}, H^{0}(\lambda)\right)\right.
$$

From now on we assume that $E_{2}^{k, l} \neq 0$. We apply Lemma 4.1 and Remark 4.4 to conclude that there exists a dominant weight $\gamma=p \delta+u \cdot 0$ with $\operatorname{Hom}_{G}\left(V(\gamma), \mathrm{H}^{l}\left(G_{1}, H^{0}(\lambda)\right)^{(-1)}\right) \neq 0$ and $\operatorname{Ext}_{G}^{k}\left(V(\lambda)^{(r-1)}, H^{0}(\gamma)\right) \neq 0$. Furthermore, by Propositions 4.2 and 4.3 there exists a sequence of non-zero weights $\lambda=\gamma_{0}, \gamma_{1}, \ldots, \gamma_{r-1}, \gamma_{r}=\lambda \in X(T)_{+}$with $\gamma_{1}=\gamma$ such that $\gamma_{j}=p \delta_{j}+u_{j} \cdot 0$ for some $u_{j} \in W$ and nonzero $\delta_{j} \in X(T)_{+}$. In addition,

$$
k+l \geq\left(\sum_{j=1}^{r}(p-1)\left\langle\delta_{j}, \tilde{\alpha}^{\vee}\right\rangle\right)-r .
$$

Equality requires that $p \delta_{j}-\delta_{j-1}+u_{j} \cdot 0=\left(\left(l_{j}-l\left(u_{j-1}\right)\right) / 2\right) \tilde{\alpha}$ and that $\left\langle-u_{j} \cdot 0, \tilde{\alpha}^{\vee}\right\rangle=\ell\left(u_{j}\right)+1$ for all $1 \leq j \leq r$, where $l_{j}$ is as in Proposition 4.2. It follows immediately that $E_{2}^{k, l}=0$ whenever $k+l<r(p-2)$.

Looking at $\left.\operatorname{Hom}_{G}\left(V(\gamma), \mathrm{H}^{l}\left(G_{1}, H^{0}(\lambda)\right)^{(-1)}\right) \cong \operatorname{Hom}_{B}\left(V(\gamma), S^{\frac{l-2 n+1}{2}}\left(\mathfrak{u}^{*}\right) \otimes \omega_{1}\right)\right)$ one concludes that for $l \leq p-2$, all weights $\mu$ appearing in $S^{\frac{l-2 n+1}{2}}\left(\mathfrak{u}^{*}\right) \otimes \omega_{1}$ satisfy $\left\langle\mu+\rho, \alpha_{0}^{\vee}\right\rangle<p$. Hence, $\mathrm{H}^{l}\left(G_{1}, H^{0}(\lambda)\right)^{(-1)}$ is completely reducible for $l \leq p-2$. From Sections 5.1 and 5.2 one concludes that $H^{0}(\lambda)$ appears as a summand if and only if $l=p-2$. Clearly the trivial module does not appear as a summand of $\mathrm{H}^{l}\left(G_{1}, H^{0}(\lambda)\right)^{(-1)}$. But these are the only composition factors of $\mathrm{H}^{l}\left(G_{1}, H^{0}(\lambda)\right)^{(-1)}$ that could be linked to the weight $p^{r-1} \lambda$. The linkage principle now forces $l \geq p-2$. Moreover, if $l=p-2$, the only possible choice for $\gamma$ is that $\gamma=\lambda$ and hence $E_{2}^{k, p-2} \cong \operatorname{Ext}_{G}^{k}\left(V(\lambda)^{(r-1)}, H^{0}(\lambda)\right)$.

If $k+l=r(p-2)$ then (5.4.1) becomes an equality. This forces $\gamma_{1}=\gamma=p \delta+u \cdot 0=$ $((l-l(u)) / 2) \tilde{\alpha}+\omega_{1}=(l-2 n+2) \omega_{1}, \delta_{1}=\delta=\omega_{1}$, and $\left\langle-u \cdot 0, \tilde{\alpha}^{\vee}\right\rangle=\ell(u)+1$. Using a similar argument to the one in Section 5.3 one concludes that $\gamma=p \omega_{1}+s_{\tilde{\alpha}} \cdot 0=\lambda$, which forces $l=p-2$ and $k=(r-1)(p-2)$.

To summarize, we have shown that

$$
E_{2}^{k, l} \cong \begin{cases}0 & \text { if } k+l<r(p-2) \\ 0 & \text { if } l<p-2 \\ 0 & \text { if } k+l=r(p-2) \text { and } l \neq p-2 \\ \operatorname{Ext}_{G}^{k}\left(V(\lambda)^{(r-1)}, H^{0}(\lambda)\right) & \text { if } k+l=r(p-2) \text { and } l=p-2 .\end{cases}
$$

Therefore, the $((r-1)(p-2), p-2)$-term of the $E_{2}$-page transgresses to the $E_{\infty}$-page and produces an isomorphism $\operatorname{Ext}_{G}^{r(p-2)}\left(V(\lambda)^{(r)}, H^{0}(\lambda)\right) \cong \operatorname{Ext}_{G}^{(r-1)(p-2)}\left(V(\lambda)^{(r-1)}, H^{0}(\lambda)\right)$, and the claim follows by induction.

By applying Theorem 4.4, the fact that $\lambda$ is the smallest weight in its linkage class, and Theorem 2.8(B) one obtains the following theorem.

Theorem . Suppose $\Phi$ is of type $C_{n}$ with $p>2 n$. Then

(a) $\mathrm{H}^{i}\left(G\left(\mathbb{F}_{q}\right), k\right)=0$ for $0<i<r(p-2)$;

(b) $\mathrm{H}^{r(p-2)}\left(G\left(\mathbb{F}_{q}\right), k\right) \cong k$. 
6 Type $A_{n}, n \geq 2$

Assume throughout this section that $\Phi$ is of type $A_{n}, n \geq 2$, and that $p>h=n+1$. Note that type $A_{1}$ is equivalent to type $C_{1}$ which was covered in Section 5.

6.1 An Upper Bound for $r=1$. We first construct a weight $\lambda$ with

$$
\mathrm{H}^{2 p-3}\left(G, H^{0}(\lambda) \otimes H^{0}\left(\lambda^{*}\right)^{(1)}\right) \neq 0 .
$$

Set $w:=s_{\tilde{\alpha}}=s_{1} s_{2} \ldots s_{n-1} s_{n} s_{n-1} \ldots s_{2} s_{1} \in W$, where $s_{i}$ is again the reflection corresponding to the $i$ th simple root $\alpha_{i}$. Then $\ell(w)=2 n-1$ and $-w \cdot 0=n \tilde{\alpha}$. When decomposed uniquely into a sum of distinct positive roots, $-w \cdot 0$ consists of precisely all positive roots which contain either an $\alpha_{1}$ or an $\alpha_{n}$ (or both). Set $\lambda:=p \tilde{\alpha}+w \cdot 0=(p-n) \tilde{\alpha}$ and $\mu:=\tilde{\alpha}$. Then $\lambda-\mu=(p-n-1) \tilde{\alpha}$ is a weight of $S^{p-n-1}\left(\mathfrak{u}^{*}\right)$. Indeed, it is the highest weight corresponding to taking $(p-n-1)$-copies of $\phi_{\tilde{\alpha}} \in \mathfrak{u}^{*}$ (the root vector corresponding to $\tilde{\alpha}$ ). Similar to the argument in Section 5.1, we will show that

$$
P_{p-n-1}(u \cdot \lambda-\mu)=\left\{\begin{array}{l}
1 \text { if } u=1 \\
0 \text { else. }
\end{array}\right.
$$

We will work with the $\epsilon$-basis of $X(T)$. Rewrite $\left.u \cdot \lambda-\mu=((p-n) u-1)\left(\epsilon_{1}-\epsilon_{n+1}\right)\right)+u \cdot 0$ as $\sum_{i} c_{i} \epsilon_{i}$. In order for this expression to be a sum of positive roots, the coefficient $c_{1}$ has to be nonnegative and $c_{n+1}$ has to be less than or equal to zero. This forces $u\left(\epsilon_{1}\right)=\epsilon_{1}$ and $u\left(\epsilon_{n+1}\right)=\epsilon_{n+1}$. This forces now $u \cdot 0$ to be of the form $-\sum_{i} d_{i} \alpha_{i}$ with $d_{1}=d_{n+1}=0$. This implies that $u \cdot \lambda-\mu=(p-n-1) \tilde{\alpha}-\sum_{i} d_{i} \alpha_{i}$ can be written as a sum of $p-n-1$ positive roots if and only if $u=1$.

Proposition . Suppose $\Phi$ is of type $A_{n}$ with $n \geq 2$ and $p \geq n+2$. Let $\lambda=(p-n) \tilde{\alpha}=$ $(p-n) \omega_{1}+(p-n) \omega_{n}$. Then

(a) $\mathrm{H}^{2 p-3}\left(G, H^{0}(\lambda) \otimes H^{0}\left(\lambda^{*}\right)^{(1)}\right) \cong k$;

(b) $\mathrm{H}^{2 p-3}\left(G\left(\mathbb{F}_{p}\right), k\right) \neq 0$.

Proof. Part (a) follows from our analysis above and Proposition 3.2.

For part (b) suppose that $0 \neq \mu \in X(T)_{+}$is linked to $(p-n) \tilde{\alpha}$ and $\mathrm{H}^{i}\left(G, H^{0}(\mu) \otimes\right.$ $\left.H^{0}\left(\mu^{*}\right)^{(1)}\right) \neq 0$ for some $i>0$. As noted in Section 3.2, we necessarily have $\mu=p \delta+w \cdot 0$ for some $0 \neq \delta \in X(T)_{+}$and $w \in W$. Observe that, since $\mu$ lies in the root lattice, $\delta$ also lies in the root lattice. Therefore, $\left\langle\delta, \tilde{\alpha}^{\vee}\right\rangle \geq 2$. From Corollary 3.5(b) we get $i \geq 2(p-1)-1=$ $2 p-3$.

Note also that $(p-n) \tilde{\alpha}$ lies in the second fundamental $p$-alcove. Indeed, it is the reflection of the zero weight across the upper wall. So the only weight $\mu$ of the form $p \tilde{\alpha}+w \cdot 0$ with $\mu<\lambda$ would be the zero weight. And we know that $\mathrm{H}^{i}(G, k)=0$ for all $i>0$. Therefore, we can apply Theorem 2.8(B) to deduce the result.

6.2 An Upper Bound for $r>1$. The following result indentifies a non-zero cohomology class in degree $r(2 p-3)$. 
Lemma. Assume $n \geq 2$ and $p>n+2$. Let $\lambda=(p-n) \tilde{\alpha}=(p-n)\left(\omega_{1}+\omega_{n}\right)$. Then $\operatorname{Ext}_{G}^{r(2 p-3)}\left(V(\lambda)^{(r)}, H^{0}(\lambda)\right) \cong k$.

Proof. We use induction on $r$. If $r=1$ the assertion follows from Section 6.1. The following argument follows closely the argument in Section 5.4. Again we make use of the LHS spectral sequence

$$
E_{2}^{k, l}=\operatorname{Ext}_{G / G_{1}}^{k}\left(V(\lambda)^{(r)}, \mathrm{H}^{l}\left(G_{1}, H^{0}(\lambda)\right)\right) \Rightarrow \operatorname{Ext}_{G}^{k+l}\left(V(\lambda)^{(r)}, H^{0}(\lambda)\right),
$$

and assume that $E_{2}^{k, l} \neq 0$. We apply Lemma 4.1 and Remark 4.4 to conclude that there exists a dominant weight $\gamma=p \delta+u \cdot 0$ with $\operatorname{Hom}_{G}\left(V(\gamma), \mathrm{H}^{l}\left(G_{1}, H^{0}(\lambda)\right)^{(-1)}\right) \neq 0$ and $\operatorname{Ext}_{G}^{k}\left(V(\lambda)^{(r-1)}, H^{0}(\gamma)\right) \neq 0$. Furthermore, by Propositions 4.2 and 4.3 there exists a sequence of non-zero weights $\lambda=\gamma_{0}, \gamma_{1}, \ldots, \gamma_{r-1}, \gamma_{r}=\lambda \in X(T)_{+}$with $\gamma_{1}=\gamma$ such that $\gamma_{j}=p \delta_{j}+u_{j} \cdot 0$ for some $u_{j} \in W$ and nonzero $\delta_{j} \in X(T)_{+}$. Note that the linkage principle forces all $\delta_{j}$ to be in the root lattice. Since none of the fundamental weights are contained in the root lattice, $\left\langle\delta_{j}, \tilde{\alpha}^{\vee}\right\rangle \geq 2$. From (4.3.1), we get

$$
k+l \geq\left(\sum_{j=1}^{r}(p-1)\left\langle\delta_{j}, \tilde{\alpha}^{\vee}\right\rangle\right)-r \geq r(2 p-3) .
$$

For the first inequality in (6.2.1) to be an equality, from Proposition 4.3, we must have $p \delta_{j}-\delta_{j-1}+u_{j} \cdot 0=\left(\left(l_{j}-l\left(u_{j-1}\right)\right) / 2\right) \tilde{\alpha}$ (where $l_{j}$ is as in Proposition 4.2) and $\left\langle-u_{j} \cdot 0, \tilde{\alpha}^{\vee}\right\rangle=$ $\ell\left(u_{j}\right)+1$ for all $1 \leq j \leq r$. Further, for the second inequality to be an equality, clearly we must have $\left\langle\delta_{j}, \tilde{\alpha}^{\vee}\right\rangle=2$. It follows immediately that $E_{2}^{k, l}=0$ whenever $k+l<r(2 p-3)$.

Looking at $\left.\operatorname{Hom}_{G}\left(V(\gamma), \mathrm{H}^{l}\left(G_{1}, H^{0}(\lambda)\right)^{(-1)}\right) \cong \operatorname{Hom}_{B}\left(V(\gamma), S^{\frac{l-2 n+1}{2}}\left(\mathfrak{u}^{*}\right) \otimes \tilde{\alpha}\right)\right)$ one concludes that for $l \leq 2 p-3$ the only possible weights $\gamma$ of the form $p \delta+u \cdot 0$ with $\delta$ in the root lattice that make the above expression non-zero are $\lambda$ and zero. Clearly the trivial module does not appear as a section in a good filtration of $\mathrm{H}^{l}\left(G_{1}, H^{0}(\lambda)\right)^{(-1)}$ while $H^{0}(\lambda)$ appears only once. Namely, in the case $l=2 p-3$. The latter follows from the discussion in Section 6.1 .

The linkage principle now forces $l \geq 2 p-3$. Moreover, if $l=2 p-3$, the only possible choice is $\gamma=\lambda$, and hence $E_{2}^{k, 2 p-3} \cong \operatorname{Ext}_{G}^{k}\left(V(\lambda)^{(r-1)}, H^{0}(\lambda)\right)$.

If $k+l=r(2 p-3)$, then (6.2.1) becomes an equality. This forces $\gamma_{1}=\gamma=p \delta+u \cdot 0=$ $((l-l(u)) / 2) \tilde{\alpha}+\tilde{\alpha}$ and $\delta_{1}=\delta=\tilde{\alpha}$. The only elements $u$ of the Weyl group with $u \cdot 0$ being a multiple of $\tilde{\alpha}$ are the identity and $s_{\tilde{\alpha}}$. Now $\left\langle-u \cdot 0, \tilde{\alpha}^{\vee}\right\rangle=\ell(u)+1$ forces $\gamma=p \omega_{1}+s_{\tilde{\alpha}} \cdot 0=\lambda$, which forces $l=2 p-3$ and $k=(r-1)(2 p-3)$.

As in 5.4 it follows that

$$
E_{2}^{k, l} \cong \begin{cases}0 & \text { if } k+l<r(2 p-3) \\ 0 & \text { if } l<2 p-3 \\ 0 & \text { if } k+l=r(2 p-3) \text { and } l \neq 2 p-3 \\ \operatorname{Ext}_{G}^{k}\left(V(\lambda)^{(r-1)}, H^{0}(\lambda)\right) & \text { if } k+l=r(2 p-3) \text { and } l=2 p-3 .\end{cases}
$$

Therefore, the $((r-1)(p-2), p-2)$-term of the $E_{2}$-page transgresses to the $E_{\infty}$-page and produces an isomorphism $\operatorname{Ext}_{G}^{r(p-2)}\left(V(\lambda)^{(r)}, H^{0}(\lambda)\right) \cong \operatorname{Ext}_{G}^{(r-1)(p-2)}\left(V(\lambda)^{(r-1)}, H^{0}(\lambda)\right)$, and the claim follows by induction. 
Remark . We have actually shown a stronger statement. Namely, for any dominant weight $\lambda$ of the form $p \delta+u \cdot 0$ with $\delta$ in the root lattice, one has

$$
\operatorname{Ext}_{G}^{i}\left(V(\lambda)^{(r)}, H^{0}(\lambda)\right) \cong \begin{cases}0 & \text { if } i<2 p-3 \\ 0 & \text { if } i=r(2 p-3) \text { and } \lambda \neq(p-n)\left(\omega_{1}+\omega_{n}\right), \\ k & \text { if } i=r(2 p-3) \text { and } \lambda=(p-n)\left(\omega_{1}+\omega_{n}\right) .\end{cases}
$$

From Theorem 2.8(B) one concludes the following.

Corollary . Suppose $\Phi$ is of type $A_{n}$ with $n \geq 2$ and $p \geq n+2$. Then $\mathrm{H}^{r(2 p-3)}\left(G\left(\mathbb{F}_{q}\right), k\right) \neq 0$.

Corollary 6.2 and Theorem 4.4 imply that the least positive $i$ with $\mathrm{H}^{i}\left(G\left(\mathbb{F}_{q}\right), k\right) \neq 0$ satisfies $r(p-2) \leq i \leq r(2 p-3)$. In the following sections, we identify precisely the value of $i$. The answer will depend on the relationship between $p$ and $n$.

6.3 Counting Simple Roots. Let $\alpha_{1}, \alpha_{2}, \ldots, \alpha_{n}$ denote the simple roots and $\omega_{1}, \omega_{2}, \ldots, \omega_{n}$ the corresponding fundamental weights. Any weight $\gamma$ can be written in the form $\gamma=$ $\sum_{j=1}^{n} c_{j} \alpha_{j}$ with $c_{j} \in \mathbb{Q}$. We define $M_{j}(\gamma):=c_{j}, M(\gamma):=\max \left\{c_{j}\right\}$, and $m(\gamma):=\max \left\{j \mid c_{j}=\right.$ $M(\gamma)\}$. In addition we set $N_{j}:=j(n+1-j)$. We make the following

Observation . For $1 \leq j \leq n$ and $w \in W$,

(a) $\omega_{j}=\frac{1}{n+1}\left(\right.$ sum of all positive roots which contain $\alpha_{j}$ ),

(b) $N_{j}$ is the number of positive roots in $\Phi$ which contain $\alpha_{j}$,

(c) $m\left(\omega_{j}\right)=j$,

(d) $M_{m\left(\omega_{j}\right)}\left(\omega_{j}\right)=M_{j}\left(\omega_{j}\right)=M\left(\omega_{j}\right)=\frac{N_{j}}{n+1}$,

(e) $M_{j}(2 \rho)=N_{j}$,

(f) $M_{j}(2 \rho)=M_{j}\left(-w_{0} \cdot 0\right) \geq M_{j}(-w \cdot 0)$.

Suppose we have dominant weights $\lambda, \mu$ with $\lambda=p \delta_{2}+w_{2} \cdot 0, \mu=p \delta_{1}+w_{1} \cdot 0$ and $\mathrm{H}^{i}\left(G, H^{0}(\mu) \otimes H^{0}\left(\lambda^{*}\right)^{(1)}\right) \neq 0$ for some $i>0$. As in the proof of Proposition 3.4, $\lambda-\delta_{1}$ must be a weight of $S^{\frac{i-\ell\left(w_{1}\right)}{2}}\left(\mathfrak{u}^{*}\right)$. Hence $M_{m\left(\delta_{2}\right)}\left(\lambda-\delta_{1}\right) \leq \frac{i-\ell\left(w_{1}\right)}{2}$. Using $\lambda=p \delta_{2}+w_{2} \cdot 0$, it follows that

$$
2 p M_{m\left(\delta_{2}\right)}\left(\delta_{2}\right)+2 M_{m\left(\delta_{2}\right)}\left(w_{2} \cdot 0\right)-2 M_{m\left(\delta_{2}\right)}\left(\delta_{1}\right)+l\left(w_{1}\right) \leq i .
$$

Note that $M_{m\left(\delta_{2}\right)}\left(\delta_{1}\right) \leq M\left(\delta_{1}\right)=M_{m\left(\delta_{1}\right)}\left(\delta_{1}\right)$ and that $-M_{m\left(\delta_{1}\right)}\left(w_{1} \cdot 0\right) \leq l\left(w_{1}\right)$ (from Observation 2.2(B)). One obtains

$$
2 p M_{m\left(\delta_{2}\right)}\left(\delta_{2}\right)+2 M_{m\left(\delta_{2}\right)}\left(w_{2} \cdot 0\right)-2 M_{m\left(\delta_{1}\right)}\left(\delta_{1}\right)-M_{m\left(\delta_{1}\right)}\left(w_{1} \cdot 0\right) \leq i .
$$

Next assume that $\mu=\lambda$. Then (6.3.2) results in $2(p-1) M_{m\left(\delta_{1}\right)}\left(\delta_{1}\right)+M_{m\left(\delta_{1}\right)}\left(w_{1} \cdot 0\right) \leq i$. Using Observation (f) gives

$$
2(p-1) M_{m\left(\delta_{1}\right)}\left(\delta_{1}\right)+M_{m\left(\delta_{1}\right)}(-2 \rho) \leq i .
$$

Suppose now that $\delta_{1}=\omega_{j}$ for $1 \leq j \leq n$. From Observations (d) and (e), (6.3.3) becomes

$$
2\left(\frac{p-1}{n+1}\right) N_{j}-N_{j} \leq i
$$


However, we can say more than this. Suppose that in fact $M_{j}\left(-w_{1} \cdot 0\right)=N_{j}$. Then, when expressed as a sum of distinct positive roots, $-w_{1} \cdot 0$ contains all $N_{j}$ roots containing $\alpha_{j}$ and possibly some other positive roots. In other words, $-w_{1} \cdot 0=(n+1) \omega_{j}+\sigma$ where $\sigma$ is a sum of distinct positive roots not containing $\alpha_{j}$. Then

$$
\lambda=p \omega_{j}+w_{1} \cdot 0=p \omega_{j}-(n+1) \omega_{j}-\sigma=(p-n-1) \omega_{j}-\sigma \leq(p-n-1) \omega_{j} .
$$

Hence, the only way $\lambda$ can be dominant is if $\sigma=0$. In other words, $\lambda=(p-n-1) \omega_{j}$ and we have shown the following.

Proposition . Suppose that $\Phi$ is of type $A_{n}$ with $n \geq 2$ and $\lambda=p \omega_{j}+w \cdot 0 \in X(T)_{+}$for $w \in W$ is a weight of $S^{\frac{i-\ell(w)}{2}}\left(\mathfrak{u}^{*}\right) \otimes \omega_{j}$. Then

$$
i \geq\left[2\left(\frac{p-1}{n+1}\right)-1\right] j(n+1-j)
$$

with equality possible if and only if $\lambda=(p-n-1) \omega_{j}$.

Remark . The assumption that $\lambda=p \omega_{j}+w \cdot 0$ is a weight of $S^{\frac{i-\ell(w)}{2}}\left(\mathfrak{u}^{*}\right) \otimes \omega_{j}$ places restrictions on $p$ (and $n$ ). Indeed, $\lambda-\omega_{j}=(p-1) \omega_{j}+w \cdot 0$ must lie in the root lattice. But $w \cdot 0$ lies in the root lattice. Therefore, $(p-1) \omega_{j}$ must also lie in the root lattice. However, for $1 \leq j \leq(n+1) / 2$, to have $(p-1) \omega_{j}$ or symmetrically $(p-1) \omega_{n+1-j}$ in the root lattice, we must have that $(n+1)$ divides $(p-1) j$.

Given the constraints noted in the remark, it is useful to rewrite the right hand side of the proposition as

$$
\left[2\left(\frac{p-1}{n+1}\right)-1\right] j(n+1-j)=[2(p-1)-(n+1)] j\left(1-\frac{j}{n+1}\right) .
$$

6.4 Larger Weights. In this section, we will see that the only non-zero non-fundamental dominant weight $\lambda$ which can have $\mathrm{H}^{i}\left(G, H^{0}(\lambda) \otimes H^{0}\left(\lambda^{*}\right)^{(1)}\right) \neq 0$ for $0 \leq i \leq 2 p-3$ is the weight $\lambda=(p-n) \tilde{\alpha}$ considered in Section 6.1. Indeed, observe that when $p \geq n+2$, $2(p-1)+2(n-1)\left[\frac{p-1}{n+1}-1\right]>2 p-3$.

Proposition . Suppose that $\Phi$ is of type $A_{n}$ with $n \geq 2$ and $p \geq n+2$. Let $\lambda=p \mu+w \cdot 0 \in$ $X(T)_{+}$for $w \in W$ with $\langle\mu, \tilde{\alpha}\rangle \geq 2$. If $\lambda \neq(p-n) \tilde{\alpha}$, then $\mathrm{H}^{i}\left(G, H^{0}(\lambda) \otimes H^{0}\left(\lambda^{*}\right)^{(1)}\right)=0$ for

$$
0<i \leq 2(p-1)+2(n-1)\left[\frac{p-1}{n+1}-1\right] .
$$

Proof. Let $\lambda$ and $\mu$ be as given. Assume that $i \neq 0$ and $\mathrm{H}^{i}\left(G, H^{0}(\lambda) \otimes H^{0}\left(\lambda^{*}\right)^{(1)}\right) \neq 0$. Using our definition of $m(\mu)$ from Section 6.3 we write $\mu=a \omega_{m(\mu)}+\sigma$, where $\sigma$ is a sum of fundamental weights other than $\omega_{m(\mu)}$ and $a$ is a positive integer. Note that $\sigma$ is nonzero if $a=1$. Set $j=\min \{m(\mu), n+1-m(\mu)\}$. Note that $M_{m(\mu)}\left(\omega_{l}\right) \geq \frac{j}{n+1}$ for any $l \neq j$. We 
obtain the following inequality

$$
M_{m(\mu)}(\mu) \geq \begin{cases}\frac{2 N_{j}}{n+1} & \text { if } a \geq 2, \\ \frac{N_{j}+j}{n+1} & \text { else. }\end{cases}
$$

Substituting the above into equation (6.3.3) with $\delta_{1}=\mu$ yields

$$
i \geq \begin{cases}2\left(\frac{p-1}{n+1}\right)\left(2 N_{j}\right)-N_{j} & \text { if } a \geq 2, \\ 2\left(\frac{p-1}{n+1}\right)\left(N_{j}+j\right)-N_{j} & \text { else. }\end{cases}
$$

As a function of $j, N_{j}$ is increasing on the interval $(0,(n+1) / 2)$. Therefore both of the above expressions are minimal when $j$ is as small as possible. In the first case $j=1$ is possible. However, since we are assuming that $\mu \neq \omega_{1}+\omega_{n}$, we may assume that $j \geq 2$ for the second case. One obtains

$$
i \geq \begin{cases}2\left(\frac{p-1}{n+1}\right) 2 n-n & \text { if } a \geq 2, \\ 2\left(\frac{p-1}{n+1}\right)(2(n-1)+2)-2(n-1)=2\left(\frac{p-1}{n+1}\right) 2 n-2(n-1) & \text { else. }\end{cases}
$$

Hence,

$$
\begin{aligned}
i & \geq 4 n\left(\frac{p-1}{n+1}\right)-2(n-1) \\
& =4(n+1)\left(\frac{p-1}{n+1}\right)-4\left(\frac{p-1}{n+1}\right)-2(n-1) \\
& =2(p-1)+[2(n+1)-4]\left(\frac{p-1}{n+1}\right)-2(n-1) \\
& =2(p-1)+2(n-1)\left(\frac{p-1}{n+1}\right)-2(n-1) \\
& =2(p-1)+2(n-1)\left[\frac{p-1}{n+1}-1\right] .
\end{aligned}
$$

To determine sharp vanishing bounds, we need to consider the relationship between $p$ and $n$. This will be done in the succeeding sections.

6.5 The Case: $\operatorname{gcd}(p-1, n+1)=1$. It follows from Remark 6.3 that under this assumption, the weight $(p-1) \omega_{j}$ does not lie in the root lattice for any $j$. Therefore, $\mu$ must be the sum of at least two (not necessarily distinct) fundamental dominant weights, and $\left\langle\mu, \tilde{\alpha}^{\vee}\right\rangle \geq 2$. From Corollary 3.5(b), we conclude that $i \geq 2 p-3$. Combining this with Proposition 6.1, Proposition 6.4, and Theorem 2.8(A), we obtain these sharp bounds.

Theorem . Suppose $\Phi$ is of type $A_{n}$ with $n \geq 2, p \geq n+2$ and $\operatorname{gcd}(p-1, n+1)=1$. Then (a) $\mathrm{H}^{i}\left(G\left(\mathbb{F}_{p}\right), k\right)=0$ for $0<i<2 p-3$; 
(b) $\mathrm{H}^{2 p-3}\left(G\left(\mathbb{F}_{p}\right), k\right) \cong k$.

6.6 The Case: $1<\operatorname{gcd}(p-1, n+1)<n+1$. For convenience, set $g:=\operatorname{gcd}(p-1, n+1)$. We investigate here the inequality in Proposition 6.3. Note that since $n+1$ does not divide $p-1$, neither $(p-1) \omega_{1}$ nor $(p-1) \omega_{n}$ lie in the root lattice. So we restrict attention to $\omega_{j}$ with $1<j<n$. As such, there is nothing to consider unless $n \geq 3$. Without a loss of generality assume that $j \leq(n+1) / 2$. Furthermore, from Remark 6.3 , we may assume that $j(p-1)$ is divisible by $(n+1)$.

Consider the function $f(j)=\left[2\left(\frac{p-1}{n+1}\right)-1\right] j(n+1-j)$, which is a quadratic polynomial in the variable $j$. For our purposes, we want to minimize $f(j)$. This evidently occurs when $j$ is minimal (for $j \leq(n+1) / 2$ ). So we consider the case that $j$ is minimal such that $j(p-1)$ is divisible by $(n+1)$. This implies that, $n+1=g j$, where $g=\operatorname{gcd}(n+1, p-1)$. With this subsitution, using (6.3.5), the inequality in Proposition 6.3 may be rewritten as

$$
i \geq[2(p-1)-(n+1)] j\left(1-\frac{1}{g}\right) .
$$

Suppose first that $n+1=g j$ is odd (and $n \geq 4$ ). Then, both $g$ and $j$ must be odd. Therefore, $g \geq 3$ and $3 \leq j \leq(n+1) / 2$. Hence,

$$
j\left(1-\frac{1}{g}\right) \geq 3\left(1-\frac{1}{3}\right)=2 .
$$

Equation (6.6.1) allows us to conclude that

$$
\begin{aligned}
i & \geq 2[2(p-1)-(n+1)] \\
& =4(p-1)-2(n+1) \\
& =2(p-1)+2(p-1)-2(n+1) \\
& =2(p-1)+2(p-n-2) \\
& \geq 2(p-1)=2 p-2
\end{aligned}
$$

since $p \geq n+2$. So we get a bound on $i$ which is strictly larger than $2 p-3$.

Consider now the case that $n+1=g j$ is even (and $n \geq 3$ ). Since $p-1$ is even, $g$ is necessarily even. In particular, $g \geq 2$. Since $g \neq n+1$, we also have $j \geq 2$. Suppose first that $j \geq 4$. Then

$$
j\left(1-\frac{1}{g}\right) \geq 4\left(1-\frac{1}{2}\right)=2 .
$$

And so the same argument as in the $n+1$-odd case would show that $i \geq 2 p-2$.

Suppose next that $j=3$ and $g>2$. Then $g \geq 4$. Then,

$$
j\left(1-\frac{1}{g}\right) \geq 3\left(1-\frac{1}{4}\right)=\frac{9}{4}>2 .
$$

We conclude that $i>2 p-2$. 
Suppose next that $j=3$ and $g=2$. Then $n+1=6$ and $p-1>n+1=6$. So $p \geq 11$ (as $p$ is prime). Here we get

$$
\begin{aligned}
i & \geq[2(p-1)-(n+1)] j\left(1-\frac{1}{g}\right) \\
& =[2(p-1)-6] 3\left(1-\frac{1}{2}\right) \\
& =3[(p-1)-3] \\
& =3 p-12 \\
& =2 p-3+p-9 \\
& \geq 2 p-1
\end{aligned}
$$

since $p \geq 11$.

Suppose next that $j=2$, i.e., $n+1=2 g$. Since $p-1>n+1$ and $g$ divides $p-1$, we must have $p-1 \geq 3 g$. Write $p-1=(3+m) g$ for an integer $m \geq 0$. Here we get

$$
\begin{aligned}
i & \geq[2(p-1)-(n+1)] j\left(1-\frac{1}{g}\right) \\
& =[2(p-1)-2 g] 2\left(1-\frac{1}{g}\right) \\
& =4(p-1)-\frac{4(p-1)}{g}-4 g+4 \\
& =2(p-1)+2(p-1)-\frac{4(p-1)}{g}-4 g+4 \\
& =2(p-1)+2(3+m) g-4(3+m)-4 g+4 \\
& =2(p-1)+2 g-8+m(2 g-4) \\
& \geq 2(p-1)+2 g-8
\end{aligned}
$$

since $m \geq 0$ and $g \geq 2$. If $g \geq 4$, then we conclude that $i \geq 2 p-2$.

However, if $g=2$, we can only conclude that $i \geq 2 p-6$. This happens when $n+1=g j=4$ or $n=3$. Note that for $n=3$, we either have $g=2$ or $g=4$ with the latter case falling into the $n+1$ divides $p-1$ category. The case of $n=3$ will be dealt with specifically in Section 6.11. We summarize our findings in the following proposition.

Proposition . Suppose $\Phi$ is of type $A_{n}$ with $n \geq 4$. Suppose further that $p>n+2$ and $1<\operatorname{gcd}(p-1, n+1)<n+1$. Let $\lambda=p \omega_{j}+w \cdot 0 \in X(T)+$ for $2 \leq j \leq n-1$ and $w \in W$. Then $\mathrm{H}^{i}\left(G, H^{0}(\lambda) \otimes H^{0}\left(\lambda^{*}\right)^{(1)}\right)=0$ for $0<i \leq 2 p-3$.

6.7 The Case: $p-1=n+1$. Under this condition, we can explicitly construct a weight $\lambda$ with $\mathrm{H}^{p-2}\left(G, H^{0}(\lambda) \otimes H^{0}\left(\lambda^{*}\right)^{(1)}\right) \neq 0$. Let $\lambda=p \omega_{1}+w \cdot 0$ where $w=s_{1} s_{2} s_{3} \ldots s_{n}$ with $s_{i}$ denoting the simple reflection corresponding to the $i$ th simple root. Then $-w \cdot 0$ is the sum of all $n$ positive roots containing $\alpha_{1}$. In other words, $-w \cdot 0=(n+1) \omega_{1}$. So 
$\lambda=p \omega_{1}+w \cdot 0=p \omega_{1}-(n+1) \omega_{1}=\omega_{1}$. Since $\ell(w)=n=p-2$, by Proposition 3.2,

$$
\operatorname{dim} \mathrm{H}^{p-2}\left(G, H^{0}\left(\omega_{1}\right) \otimes H^{0}\left(\omega_{1}^{*}\right)^{(1)}\right)=\sum_{u \in W}(-1)^{\ell(u)} P_{0}\left(u \cdot \omega_{1}-\omega_{1}\right)=1 .
$$

Note that $\omega_{1}^{*}=\omega_{n}$ and one can similarly argue that $H^{p-2}\left(G, H^{0}\left(\omega_{n}\right) \otimes H^{0}\left(\omega_{n}^{*}\right)^{(1)}\right) \cong k$.

Theorem . Suppose $\Phi$ is of type $A_{n}$ with $n \geq 2$ and $p-1=n+1$. Then

(a) $\mathrm{H}^{i}\left(G\left(\mathbb{F}_{p}\right), k\right)=0$ for $0<i<p-2$;

(b) $\mathrm{H}^{p-2}\left(G\left(\mathbb{F}_{p}\right), k\right) \cong k \oplus k$.

Proof. Part (a) follows from Theorem 4.4. For part (b), from the discussion above, we know that $\mathrm{H}^{p-2}\left(G, H^{0}(\lambda) \otimes H^{0}\left(\lambda^{*}\right)^{(1)}\right) \cong k$ for $\lambda=\omega_{1}$ or $\lambda=\omega_{n}$. We claim that if $\mathrm{H}^{p-2}\left(G, H^{0}(\lambda) \otimes H^{0}\left(\lambda^{*}\right)^{(1)}\right) \neq 0$ for a dominant weight $\lambda$, then $\lambda=\omega_{1}$ or $\omega_{n}$. To see this, write $\lambda=p \mu+w \cdot 0$ for some $\mu \in X(T)_{+}$and $w \in W$. By Corollary 3.5(b), $\mu$ must be a fundamental dominant weight. Apply Proposition 6.3 with $p-1=n+1$. The proposition gives that, for $1<j<n$,

$$
i \geq j(n+1-j)=j(p-1-j) \geq 2(p-1-2)=2 p-6=(p-2)+(p-4) .
$$

For $n \geq 3, p=n+2 \geq 5$ and so this gives $i>p-2$. Hence, $\mu=\omega_{1}$ or $\omega_{n}$. In other words $\lambda=p \omega_{1}+w \cdot 0$ or $\lambda=p \omega_{n}+w \cdot 0$, respectively. From the proof of Corollary 3.5, we observe that in order to have $\mathrm{H}^{p-2}\left(G, H^{0}(\lambda) \otimes H^{0}\left(\lambda^{*}\right)^{(1)}\right) \neq 0$, when $-w \cdot 0$ is expressed as a sum of distinct positive roots, one of those roots must be $\tilde{\alpha}=\alpha_{1}+\alpha_{2}+\cdots+\alpha_{n}$. From Observation 2.2(B), it follows that $\ell(w) \geq n=p-2$. Applying Proposition 3.2, we see that

$$
\operatorname{dim} \mathrm{H}^{p-2}\left(G, H^{0}(\lambda) \otimes H^{0}\left(\lambda^{*}\right)^{(1)}\right)=\sum_{u \in W}(-1)^{\ell(u)} P_{0}(u \cdot \lambda-\mu) .
$$

One has non-zero cohomology only if $u \cdot \lambda-\mu=0$ which can only happen if $\lambda=\mu$, which gives the claim.

Since the only dominant weight less than $\omega_{1}$ or $\omega_{n}$ is the zero weight, $\mathrm{H}^{i}(G, k)=0$ for $i>0$, and $\omega_{1}$ and $\omega_{n}$ lie in different linkage classes, the discussion in Section 2.8 gives part (b).

6.8 The Case: $\operatorname{gcd}(p-1, n+1)=n+1<p-1$. The case $p-1=n+1$ is excluded since that was dealt with in Section 6.7. Since $n+1$ divides $p-1,(p-1) \omega_{j}$ lies in the root lattice for all $1 \leq j \leq n$, and we need to allow $\lambda=p \omega_{j}+w \cdot 0$ for all $j$ in our general argument.

Write $p-1=d(n+1)$ for an integer $d \geq 2$. We can rewrite the inequality in Proposition 6.3 (see also (6.3.5) ) as

$$
\begin{aligned}
i & \geq 2 j(p-1)-2 j^{2}\left(\frac{p-1}{n+1}\right)-j(n+1)+j^{2} \\
& =2(p-1)+(2 j-2)(p-1)-2 j^{2}\left(\frac{p-1}{n+1}\right)-j(n+1)+j^{2} \\
& =2(p-1)+\left[(2 j-2)(n+1)-2 j^{2}\right]\left(\frac{p-1}{n+1}\right)-j(n+1)+j^{2} \\
& =2(p-1)+\left[(2 j-2)(n+1)-2 j^{2}\right] d-j(n+1)+j^{2} .
\end{aligned}
$$


For $j=1$ (or $j=n$ ), this inequality allows for a value of $i<2 p-3$. This will be discussed more in the next section. For this section, we focus on the case $2 \leq j \leq n-1$. By default, we need $n \geq 3$. For such $j$, the least value of the right hand side above occurs when $j=2$ (or $j=n-1$ ). Substituting $j=2$, the above inequality becomes

$$
i \geq 2(p-1)+(2 n-6) d-2 n+2 .
$$

Since $d \geq 2$, (6.8.1) becomes

$$
i \geq 2(p-1)+2 n-10 .
$$

If $n \geq 5$, then $i \geq 2 p-2$. If $n=4$ with $d \geq 3$, then (6.8.1) becomes

$$
i \geq 2(p-1)+(2 * 4-6) 3-2 * 4+2=2 p-2 .
$$

If $n=4$ and $d=2$, then $p-1=2(4+1)=10$ or $p=11$ and we can only say that $i \geq 2 p-6$. This case will be considered in Section 6.12. For $n=3$, notice that the value of $d$ is irrelevant in (6.8.1). Irrespective of $d$, we conclude that $i \geq 2 p-6$. This case will be discussed in Section 6.11. We summarize the conclusions of this section in the following.

Proposition . Suppose $\Phi$ is of type $A_{n}$ with $n \geq 4$. Suppose further that $p>n+2$ and $\operatorname{gcd}(p-1, n+1)=n+1$. If $n=4$, assume further that $p \neq 11$. Let $\lambda=p \omega_{j}+w \cdot 0 \in X(T)_{+}$ for $2 \leq j \leq n-1$ and $w \in W$. Then $\mathrm{H}^{i}\left(G, H^{0}(\lambda) \otimes H^{0}\left(\lambda^{*}\right)^{(1)}\right)=0$ for $0<i \leq 2 p-3$.

6.9 The Case: $\operatorname{gcd}(p-1, n+1)=n+1<p-1$, continued. In this section we investigate the case of $\lambda=p \omega_{1}+w \cdot 0$ (or symmetrically, $\lambda=p \omega_{n}+w \cdot 0$ ).

Lemma . Suppose that $\Phi$ is of type $A_{n}$ Let $\lambda=p \omega_{1}+w \cdot 0 \in X(T)_{+}$or $\lambda=p \omega_{n}+$ $w \cdot 0 \in X(T)_{+}$and $\mu=p \omega_{j}+v \cdot 0 \in X(T)_{+}$for $1 \leq j \leq n$ and $v, w \in W$. Then $\mathrm{H}^{i}\left(G, H^{0}(\mu) \otimes H^{0}\left(\lambda^{*}\right)^{(1)}\right)=0$ for $0<i<(p-1-n) n-(n+1-j) j+\ell(v)$.

Proof. We give the argument for $\omega_{1}$. An analogous argument works for $\omega_{n}$. If $\lambda=p \omega_{1}+w \cdot 0$ is dominant, then a direct computation shows that $w=s_{k} s_{k-1} \ldots s_{1}$ and $\lambda=(p-k-1) \omega_{1}+$ $\omega_{k+1}$, where $0 \leq k \leq n$. Here we are using the conventions $s_{0}=1$ and $\omega_{n+1}=0$. Moreover, we have the following equations for the formal characters

$$
\operatorname{char}\left(V\left((p-k) \omega_{1}\right) \otimes V\left(\omega_{k}\right)\right)=\operatorname{char} V\left((p-k) \omega_{1}+\omega_{k}\right)+\operatorname{char} V\left((p-k-1) \omega_{1}+\omega_{k+1}\right) .
$$

As discussed earlier, the module $\operatorname{ind}_{B}^{G}\left(S^{m}\left(\mathfrak{u}^{*}\right) \otimes \omega_{j}\right)$ has a good filtration. We conclude that

$$
\begin{aligned}
& \operatorname{dim} \operatorname{Hom}_{G}\left(V\left(p \omega_{1}+w \cdot 0\right), \operatorname{ind}_{B}^{G}\left(S^{m}\left(\mathfrak{u}^{*}\right) \otimes \omega_{j}\right)\right) \\
& \leq \operatorname{dim} \operatorname{Hom}_{G}\left(V\left((p-k) \omega_{1}\right) \otimes V\left(\omega_{k}\right), \operatorname{ind}_{B}^{G}\left(S^{m}\left(\mathfrak{u}^{*}\right) \otimes \omega_{j}\right)\right) \\
& =\operatorname{dim} \operatorname{Hom}_{G}\left(V\left((p-k) \omega_{1}\right), \operatorname{ind}_{B}^{G}\left(S^{m}\left(\mathfrak{u}^{*}\right) \otimes \omega_{j}\right) \otimes H^{0}\left(\omega_{n+1-k}\right)\right),
\end{aligned}
$$

where $k=\ell(w)$.

Note that $\left\langle\omega_{j}-u\left(\omega_{k}\right), \alpha_{l}^{\vee}\right\rangle \geq-1$ for all $u \in W$ and $1 \leq l \leq n$. It follows from [KLT] that $R^{j} \operatorname{ind}_{B}^{G}\left(S^{m}\left(\mathfrak{u}^{*}\right) \otimes\left(\omega_{j}+u\left(\omega_{n+1-k}\right)\right)\right)=0$ for $j>0$. Using the long exact sequence that one 
ON THE VANISHING RANGES FOR THE COHOMOLOGY OF FINITE GROUPS OF LIE TYPE 25 obtains from a $B$-filtration of $H^{0}\left(\omega_{n+1-k}\right)$ one concludes that

$$
\begin{aligned}
& \operatorname{dim} \operatorname{Hom}_{G}\left(V\left(p \omega_{1}+w \cdot 0\right), \operatorname{ind}_{B}^{G}\left(S^{m}\left(\mathfrak{u}^{*}\right) \otimes \omega_{j}\right)\right) \\
& \leq \operatorname{dim} \operatorname{Hom}_{G}\left(V\left((p-k) \omega_{1}\right), \operatorname{ind}_{B}^{G}\left(S^{m}\left(\mathfrak{u}^{*}\right) \otimes \omega_{j}\right) \otimes H^{0}\left(\omega_{n+1-k}\right)\right) \\
& =\frac{1}{\left|\operatorname{Stab}_{W}\left(\omega_{k}\right)\right|} \sum_{u \in W} \operatorname{dim} \operatorname{Hom}_{G}\left(V\left((p-k) \omega_{1}\right), \operatorname{ind}_{B}^{G}\left(S^{m}\left(\mathfrak{u}^{*}\right) \otimes \omega_{j}-u\left(\omega_{k}\right)\right)\right) .
\end{aligned}
$$

The Weyl module $V\left((p-k) \omega_{1}\right)$ has one-dimensional weight spaces [J1, II 2.16]. A theorem of Kostant [Hum1, 24.2] implies that

$$
\sum_{m \geq 0} \sum_{x \in W}(-1)^{\ell(x)} P_{m}\left(x \cdot\left((p-k) \omega_{1}\right)-\omega_{j}+u\left(\omega_{k}\right)\right)=1 .
$$

According to [AJ, 3.8] and [KLT] we know that

$$
\begin{aligned}
& \sum_{x \in W}(-1)^{\ell(x)} P_{m}\left(x \cdot\left((p-k) \omega_{1}\right)-\omega_{j}+u\left(\omega_{k}\right)\right) \\
& =\operatorname{dim} \operatorname{Hom}_{G}\left(V\left((p-k) \omega_{1}\right), \operatorname{ind}_{B}^{G}\left(S^{m}\left(\mathfrak{u}^{*}\right) \otimes \omega_{j}-u\left(\omega_{k}\right)\right) \geq 0,\right.
\end{aligned}
$$

for all $m \geq 0$. Clearly, for $x \neq 1$,

$$
\operatorname{height}\left((p-k) \omega_{1}-\omega_{j}+u\left(\omega_{k}\right)\right)>\operatorname{height}\left(x \cdot\left((p-k) \omega_{1}\right)-\omega_{j}+u\left(\omega_{k}\right)\right) \text {. }
$$

We conclude that

$$
\sum_{x \in W}(-1)^{\ell(x)} P_{m}\left(x \cdot\left((p-k) \omega_{1}\right)-\omega_{j}+u\left(\omega_{k}\right)\right)=\left\{\begin{array}{l}
1 \text { if } m=\operatorname{height}\left((p-k) \omega_{1}-\omega_{j}+u\left(\omega_{k}\right)\right) \\
0 \text { else. }
\end{array}\right.
$$

We have

$$
\begin{aligned}
\operatorname{height}\left((p-k) \omega_{1}-\omega_{j}+u\left(\omega_{k}\right)\right) & \geq \operatorname{height}\left((p-k) \omega_{1}-\omega_{j}-\omega_{n+1-k}\right) \\
& =\frac{(p-k) n}{2}-\frac{(n+1-j) j}{2}-\frac{(n+1-k) k}{2} .
\end{aligned}
$$

Hence,

if

$$
\operatorname{dim} \operatorname{Hom}_{G}\left(V\left(p \omega_{1}+w \cdot 0\right), \operatorname{ind}_{B}^{G}\left(S^{m} \mathfrak{u}^{*} \otimes \omega_{j}\right)\right)=0,
$$

$$
m<\frac{(p-k) n-(n+1-j) j-(n+1-k) k}{2}
$$

where $k=\ell(w)$.

Setting $m=\frac{i-\ell(v)}{2}$ and applying Lemma 3.1 and (3.2.1) yields

$$
\mathrm{H}^{i}\left(G, H^{0}\left(p \omega_{1}+w \cdot 0\right) \otimes H^{0}\left(\left(p \omega_{j}+v \cdot 0\right)^{*}\right)^{(1)}\right)=0
$$

for

$$
\frac{i-\ell(v)}{2}<\frac{(p-k) n-(n+1-j) j-(n+1-k) k}{2} .
$$

Hence, one obtains vanishing for

$$
i<(p-k) n-(n+1-k) k-(n+1-j) j+\ell(v) .
$$


Since the global minimum of $(p-k) n-(n+1-k) k$ on the closed interval $[1, n]$ occurs at $k=n$, we get the claimed vanishing for

$$
i \leq(p-1-n) n-(n+1-j) j+\ell(v) .
$$

Proposition . Suppose $\Phi$ is of type $A_{n}$ with $n \geq 3$. Suppose further that $p>n+2$ and $\operatorname{gcd}(p-1, n+1)=n+1$. Let $\lambda=p \omega_{1}+w \cdot 0 \in X(T)_{+}$or $\lambda=p \omega_{n}+w \cdot 0$ with $w \in W$. Then $\mathrm{H}^{i}\left(G, H^{0}(\lambda) \otimes H^{0}\left(\lambda^{*}\right)^{(1)}\right)=0$ for $0<i \leq 2 p-3$.

Proof. Equation (6.9.1) implies, for the case $\lambda=\mu$, vanishing for $0<i<(p-k) n-(n+$ $1-k) k-n+\ell(v)=(p-k) n-(n-k) k-n$. Again the global minimum occurs at $k=n$ and one obtains vanishing for $0<i \leq(p-1-n) n$. Observe that $p \geq 2(n+1)+1=2 n+3$. Now

$$
\begin{aligned}
(p-1-n) n & =2 p-3+p(n-2)-n(n+1)+3 \\
& \geq 2 p-3+(2 n+3)(n-2)-n^{2}-n+3 \\
& =2 p-3+(n-1)^{2}-4 \\
& \geq 2 p-3
\end{aligned}
$$

with equality if and only if $n=3$ and $p=2 n+3$. This case does not occur.

6.10 The Case: $n=2$. Assume for this subsection that $\Phi$ is of type $A_{2}$ with $p>3$. From Proposition 6.1 we know that $\mathrm{H}^{2 p-3}\left(G\left(\mathbb{F}_{p}\right), k\right) \neq 0$. Proposition 6.5 implies that $2 p-3$ is indeed the lowest bound unless 3 divides $p-1$. Note that the case $p-1=3$ is not possible for a prime $p$. If 3 divides $p-1$, then the only possible non-zero cohomology in lower degrees would come from weights of the form $\lambda=p \omega_{1}+w \cdot 0$ or the dual case $\lambda=p \omega_{2}+w \cdot 0$. It follows from Lemma 6.9 (see also the proof of Proposition 6.9) that $\mathrm{H}^{i}\left(G, H^{0}(\lambda) \otimes H^{0}\left(\lambda^{*}\right)^{(1)}\right)=0$ for $i<2 p-6$. Moreover, using the arguments of Lemma 6.9 one can show that $\mathrm{H}^{i}\left(G, H^{0}(\lambda) \otimes H^{0}\left(\lambda^{*}\right)^{(1)}\right)=0$ for $i \leq 2 p-6$ unless $\lambda=(p-3) \omega_{j}$. In that case $\sum_{u \in W}(-1)^{\ell(u)} P_{p-4}\left(u \cdot\left((p-3) \omega_{j}\right)-\omega_{j}\right)=1$ because the height of $(p-4) \omega_{j}$ is exactly $p-4$. Proposition 3.2 now says that $\mathrm{H}^{2 p-6}\left(G, H^{0}\left((p-3) \omega_{j}\right) \otimes H^{0}\left((p-3) \omega_{(3-j)}\right)^{(1)}\right) \cong k$, $j=1,2$. Note that $\omega_{1}$ and $\omega_{2}$ are in different linkage classes. We conclude the following from Theorem 2.8(A) and the linkage discussion in Section 2.8.

Proposition . Suppose $\Phi$ is of type $A_{2}$ and $p>3$.

(a) If 3 divides $p-1$, then

(i) $\mathrm{H}^{i}\left(G\left(\mathbb{F}_{p}\right), k\right)=0$ for $0<i<2 p-6$;

(ii) $\mathrm{H}^{2 p-6}\left(G\left(\mathbb{F}_{p}\right), k\right) \cong k \oplus k$.

(b) If 3 does not divide $p-1$, then $\mathrm{H}^{i}\left(G\left(\mathbb{F}_{p}\right), k\right)=0$ for $0<i<2 p-3$.

6.11 The Case: $n=3$. Let $\Phi$ be of type $A_{3}$ with $p>4$. The case $p=5$ is included in Proposition 6.7. For the remainder of this section we assume that $p>5$. 
Lemma . Suppose that $\Phi$ is of type $A_{3}$ with $p>4$. Then

$$
\sum_{u \in W}(-1)^{\ell(u)} P_{p-5}\left(u \cdot\left((p-4) \omega_{2}\right)-\omega_{2}\right)=1 .
$$

Proof. Observe that $2 \omega_{2}=\alpha_{1}+2 \alpha_{2}+\alpha_{3}=\epsilon_{1}+\epsilon_{2}-\epsilon_{3}-\epsilon_{4}$. For $u \cdot\left((p-4) \omega_{2}\right)-\omega_{2}$ to be a sum of positive roots one needs $u\left(2 \omega_{2}\right)$ to be a sum of positive roots. This is the case if and only if either $u\left(2 \omega_{2}\right)=2 \omega_{2}$ or $u\left(2 \omega_{2}\right)=\alpha_{1}+\alpha_{3}$. But we can rule out the second case because here $u \cdot\left((p-4) \omega_{2}\right)-\omega_{2}=((p-5) / 2)\left(\alpha_{1}+\alpha_{3}\right)-\alpha_{2}+u \cdot 0$. This is clearly not the sum of positive roots. It follows that we only have to consider $u \in \operatorname{Stab}_{W}\left(\omega_{2}\right)=\left\{1, s_{1}, s_{3}, s_{1} s_{3}\right\}$ and that $u \cdot\left((p-4) \omega_{2}\right)-\omega_{2}=(p-5) \omega_{2}+u \cdot 0$. A direct computation now shows that

$$
P_{p-5}\left((p-5) \omega_{2}+u \cdot 0\right)= \begin{cases}(p-5) / 2 & \text { if } u=1 \\ (p-7) / 2 & \text { if } u=s_{1}, s_{3}, s_{1} s_{3} .\end{cases}
$$

Hence, $\sum_{u \in W}(-1)^{\ell(u)} P_{p-5}\left(u \cdot\left((p-4) \omega_{2}\right)-\omega_{2}\right)=\sum_{u \in \operatorname{Stab}_{W}\left(\omega_{2}\right)}(-1)^{\ell(u)} P_{p-5}\left(u \cdot\left((p-4) \omega_{2}\right)-\right.$ $\left.\omega_{2}\right)=1$.

By Proposition 6.1 we know that $\mathrm{H}^{2 p-3}\left(G\left(\mathbb{F}_{p}\right), k\right) \neq 0$. Note that $\operatorname{gcd}(p-1, n+1)=$ $\operatorname{gcd}(p-1,4)=2$ or 4 . If $\operatorname{gcd}(p-1,4)=2$, then by Remark 6.3 , the only possible non-zero cohomology in lower degrees would come from weights of the form $\lambda=p \omega_{2}+w \cdot 0$. On the other hand, if $\operatorname{gcd}(p-1,4)=4$, then Proposition 6.9 gives the same conclusion.

Suppose $\lambda=p \omega_{2}+w \cdot 0$. It follows from Proposition 6.3 that $H^{i}\left(G, H^{0}(\lambda) \otimes H^{0}\left(\lambda^{*}\right)^{(1)}\right)=0$ for $i<2 p-6$ and that $\mathrm{H}^{2 p-6}\left(G, H^{0}(\lambda) \otimes H^{0}\left(\lambda^{*}\right)^{(1)}\right)=0$ unless $\lambda=(p-4) \omega_{2}$. That occurs if $w \cdot 0=-4 \omega_{2}$. By direct computation one finds that $\ell(w)=4$. By Proposition 3.2 and Lemma 6.11 above, we have

$$
\begin{aligned}
\operatorname{dim} H^{2 p-6}\left(G, H^{0}\left((p-4) \omega_{2}\right)\right. & \left.\otimes H^{0}\left((p-4) \omega_{2}^{*}\right)^{(1)}\right) \\
& =\sum_{u \in W}(-1)^{\ell(u)} P_{\frac{(2 p-6)-4}{2}}\left(u \cdot\left((p-4) \omega_{2}\right)-\omega_{2}\right) \\
& =\sum_{u \in W}(-1)^{\ell(u)} P_{p-5}\left(u \cdot\left((p-4) \omega_{2}\right)-\omega_{2}\right)=1 .
\end{aligned}
$$

Combining this with Theorem $2.8(\mathrm{~A})$ one obtains that $\mathrm{H}^{2 p-6}\left(G\left(\mathbb{F}_{p}\right), k\right) \cong k$. We summarize our findings for $\Phi=A_{3}$ below.

Proposition . Suppose $\Phi$ is of type $A_{3}$ and $p>4$.

(a) If $p=5$, then

(i) $\mathrm{H}^{i}\left(G\left(\mathbb{F}_{p}\right), k\right)=0$ for $0<i<p-2$;

(ii) $\mathrm{H}^{p-2}\left(G\left(\mathbb{F}_{p}\right), k\right) \cong k \oplus k$.

(b) If $p>5$, then

(i) $\mathrm{H}^{i}\left(G\left(\mathbb{F}_{p}\right), k\right)=0$ for $0<i<2 p-6$;

(ii) $\mathrm{H}^{2 p-6}\left(G\left(\mathbb{F}_{p}\right), k\right) \cong k$.

6.12 The Case: $n=4$ and $p=11$. Assume for this section that $\Phi$ is of type $A_{4}$ with $p=11$. Then $\operatorname{gcd}(p-1, n+1)=\operatorname{gcd}(10,5)=5$ and $2 p-3=19$. It follows from Proposition 6.9 and Proposition 6.4 that the only non-zero cohomology in degrees lower than 19 has to 
come from weights of the form $\lambda=11 \omega_{2}+w \cdot 0=6 \omega_{2}$ or $\lambda=11 \omega_{3}+w \cdot 0=6 \omega_{3}$. In these cases we could potentially achieve non-vanishing in degree 18. The weights $\omega_{2}$ and $\omega_{3}$ are dual. We give the argument for $\omega_{2}$. An analogous argument works for $\omega_{3}$. Here $\ell(w)=6$. According to Proposition 3.2, to have $\mathrm{H}^{18}\left(G, H^{0}\left((p-5) \omega_{2}\right) \otimes H^{0}\left((p-5) \omega_{3}\right)^{(1)}\right) \neq 0$ one needs $\sum_{u \in W}(-1)^{\ell(u)} P_{6}\left(u \cdot\left(6 \omega_{2}\right)-\omega_{2}\right) \neq 0$. The lemma below (Lemma 6.12) rules this case out.

According to Proposition $6.1, \mathrm{H}^{19}\left(G\left(\mathbb{F}_{p}\right), k\right) \neq 0$. Again it follows from Proposition 6.9 and Proposition 6.4 that any cohomology in degree 19 other then the one coming from Proposition 6.1 has to come from weights of the form $\lambda=11 \omega_{2}+w \cdot 0$ or $\lambda=11 \omega_{3}+w \cdot 0$. Suppose $\lambda=11 \omega_{2}+w \cdot 0$. Again, the $\omega_{3}$ case is analogous. Proposition 3.2 implies that cohomology in odd degrees has to come from weights with corresponding $w \in W$ of odd length. Now equation (6.3.1) shows that this can only happen if $\ell(w)=5$. Note that here $M_{2}(-w \cdot 0)=\ell(w)=5$. The only such weight is $\lambda=6 \omega_{2}+\tilde{\alpha}$. By Proposition 3.2 it suffices to show that $\sum_{u \in W}(-1)^{\ell(u)} P_{7}\left(u \cdot\left(6 \omega_{2}+\tilde{\alpha}\right)-\omega_{2}\right)=0$. We conclude from the lemma below that $\mathrm{H}^{i}\left(G, H^{0}(\lambda) \otimes H^{0}\left(\lambda^{*}\right)^{(1)}\right)=0$ for $0<i<2 p-3$ and further that $\mathrm{H}^{2 p-3}\left(G, H^{0}(\lambda) \otimes H^{0}\left(\lambda^{*}\right)^{(1)}\right)=0$ unless $\lambda=(p-n) \tilde{\alpha}=7 \tilde{\alpha}$.

Lemma . Suppose that $\Phi$ is of type $A_{4}$ with $p=11$. Then

(a) $\sum_{u \in W}(-1)^{\ell(u)} P_{6}\left(u \cdot\left(6 \omega_{2}\right)-\omega_{2}\right)=0$,

(b) $\sum_{u \in W}(-1)^{\ell(u)} P_{7}\left(u \cdot\left(6 \omega_{2}+\tilde{\alpha}\right)-\omega_{2}\right)=0$.

Proof. (a) Observe that $5 \omega_{2}=3 \alpha_{1}+6 \alpha_{2}+4 \alpha_{3}+2 \alpha_{4}=3 \epsilon_{1}+3 \epsilon_{2}-2 \epsilon_{3}-2 \epsilon_{4}-2 \epsilon_{5}$. For $u \cdot\left(6 \omega_{2}\right)-\omega_{2}$ to be a sum of positive roots one needs $u\left(5 \omega_{2}\right)$ to be a sum of positive roots. This is the case if and only if either $u\left(5 \omega_{2}\right)=5 \omega_{2}$ or $u\left(5 \omega_{2}\right)=3 \alpha_{1}+\alpha_{2}+4 \alpha_{3}+2 \alpha_{4}$. Let us consider the second case. Here $u$ is an element of the co-set $s_{2} \cdot \operatorname{Stab}_{W}\left(\omega_{2}\right)$. Note that for any $u \in s_{2} \cdot \operatorname{Stab}_{W}\left(\omega_{2}\right)$ the expression $-u \cdot 0$ contains some positive multiple of the root $\alpha_{2}$. Therefore $u \cdot\left(6 \omega_{2}\right)-\omega_{2}=3 \alpha_{1}+4 \alpha_{3}+2 \alpha_{4}+u \cdot 0$ is not a sum of positive roots. It suffices therefore to look at $\sum_{u \in \operatorname{Stab}_{W}\left(\omega_{2}\right)}(-1)^{\ell(u)} P_{6}\left(5 \omega_{2}+u \cdot 0\right)$. A straightforward but tedious calculation now shows that $\sum_{u \in \operatorname{Stab}_{W}\left(\omega_{2}\right)}(-1)^{\ell(u)} P_{6}\left(5 \omega_{2}+u \cdot 0\right)=0$.

(b) As in part (a) for $u \cdot\left(6 \omega_{2}+\tilde{\alpha}\right)-\omega_{2}$ to be the sum of positive roots one needs either $u \in \operatorname{Stab}_{W}\left(\omega_{2}\right)$ or $u \in s_{2} \cdot \operatorname{Stab}_{W}\left(\omega_{2}\right)$. However, the second case results in weights that cannot be written as sums of 7 positive roots. As in part (a) it suffices therefore to look at $\sum_{u \in \operatorname{Stab}_{W}\left(\omega_{2}\right)}(-1)^{\ell(u)} P_{7}\left(6 \omega_{2}+u \cdot \tilde{\alpha}\right)$ which can be shown to be zero.

Both parts of the lemma can also be readily checked by using computer software such as MAGMA $B \mathrm{BC}, \mathrm{BCP}$.

6.13 Summary for $r=1$. The following theorem addresses (1.1.1) and (1.1.2) for type $A_{n}$ when $r=1$.

Theorem . Suppose $\Phi$ is of type $A_{n}$ with $n \geq 2$. Suppose further that $p>n+1$.

(a) (Generic case) If $p>n+2$ and $n>3$, then

(i) $\mathrm{H}^{i}\left(G\left(\mathbb{F}_{p}\right), k\right)=0$ for $0<i<2 p-3$;

(ii) $\mathrm{H}^{2 p-3}\left(G\left(\mathbb{F}_{p}\right), k\right) \cong k$.

(b) If $p=n+2$, then 
(i) $\mathrm{H}^{i}\left(G\left(\mathbb{F}_{p}\right), k\right)=0$ for $0<i<p-2$;

(ii) $\mathrm{H}^{p-2}\left(G\left(\mathbb{F}_{p}\right), k\right) \cong k \oplus k$.

(c) If $n=2$ and 3 divides $p-1$, then

(i) $\mathrm{H}^{i}\left(G\left(\mathbb{F}_{p}\right), k\right)=0$ for $0<i<2 p-6$;

(ii) $\mathrm{H}^{2 p-6}\left(G\left(\mathbb{F}_{p}\right), k\right) \cong k \oplus k$.

(d) If $n=2$ and 3 does not divide $p-1$, then

(i) $\mathrm{H}^{i}\left(G\left(\mathbb{F}_{p}\right), k\right)=0$ for $0<i<2 p-3$;

(ii) $\mathrm{H}^{2 p-3}\left(G\left(\mathbb{F}_{p}\right), k\right) \cong k$.

(e) If $n=3$ and $p>5$, then

(i) $\mathrm{H}^{i}\left(G\left(\mathbb{F}_{p}\right), k\right)=0$ for $0<i<2 p-6$;

(ii) $\mathrm{H}^{2 p-6}\left(G\left(\mathbb{F}_{p}\right), k\right) \cong k$.

Proof. Let $\lambda=p \mu+w \cdot 0 \in X(T)_{+}$for $\mu \in X(T)_{+}$and $w \in W$. Based on the discussion in Section 2, our goal has been to determine the least $i>0$ such that

$$
\mathrm{H}^{i}\left(G, H^{0}(\lambda) \otimes H^{0}\left(\lambda^{*}\right)^{(1)}\right) \neq 0 .
$$

According to Proposition 6.1(a), we know that the weight $\lambda=p \tilde{\alpha}-n \tilde{\alpha}$ gives a non-zero cohomology class in degree $2 p-3$.

By Proposition 3.4(b), if $\left\langle\mu, \tilde{\alpha}^{\vee}\right\rangle \geq 2$, then $i \geq 2 p-3$. Hence, the only way to obtain a smaller $i$ is for $\mu$ to be a fundamental weight. That case has been dealt with in previous sections, from which parts (a)(i), (b), (c), (d)(i), and (e) follow. It remains to show parts (a)(ii) and (d)(ii). From Proposition $6.4, \lambda=p \tilde{\alpha}-n \tilde{\alpha}=p\left(\omega_{1}+\omega_{n}\right)$ is the only weight with $\mathrm{H}^{2 p-3}\left(G, H^{0}(\lambda) \otimes H^{0}\left(\lambda^{*}\right)^{(1)}\right) \neq 0$. The result follows by Proposition 6.1(a) and Theorem $2.8(\mathrm{~A})$.

6.14 Results for $r>1$. The following theorem addresses (1.1.1) and (1.1.2) for type $A_{n}$ when $r>1$ and $p>2 n-2$. For $n>3$, a generic vanishing bound of degree $r(2 p-3)$ can be observed.

For $n+2 \leq p \leq 2(n+1)$, the methods employed in this paper should allow one to obtain precise vanishing bounds. However, given the number of special cases encountered in the $r=1$ case, one would expect even more non-generic behavior for $r>1$. For example, it is easily seen that in the case $p=n+2$ non-vanishing already occurs in degree $r(p-2)$, i.e., $\mathrm{H}^{r(p-2)}\left(G\left(\mathbb{F}_{q}\right), k\right) \neq 0$. To give a complete answer many case-by-case arguments will be necessary, most of them rather lengthy and intricate. For brevity we limit ourselves here to the case where $\mathrm{p}$ is larger than twice the Coxeter number.

Theorem . Suppose $\Phi$ is of type $A_{n}$ with $n \geq 2$ and $p>2(n+1)$. Then

(a) (Generic case) If $n>3$, then

(i) $\mathrm{H}^{i}\left(G\left(\mathbb{F}_{q}\right), k\right)=0$ for $0<i<r(2 p-3)$;

(ii) $\mathrm{H}^{r(2 p-3)}\left(G\left(\mathbb{F}_{q}\right), k\right) \cong k$.

(b) If $n=2$ and 3 divides $q-1$, then

(i) $\mathrm{H}^{i}\left(G\left(\mathbb{F}_{q}\right), k\right)=0$ for $0<i<r(2 p-6)$;

(ii) $\mathrm{H}^{r(2 p-6)}\left(G\left(\mathbb{F}_{q}\right), k\right) \cong k \oplus k$.

(c) If $n=2$ and 3 does not divide $q-1$, then

(i) $\mathrm{H}^{i}\left(G\left(\mathbb{F}_{q}\right), k\right)=0$ for $0<i<r(2 p-3)$; 
(ii) $\mathrm{H}^{r(2 p-3)}\left(G\left(\mathbb{F}_{q}\right), k\right) \cong k$.

(d) If $n=3$, then

(i) $\mathrm{H}^{i}\left(G\left(\mathbb{F}_{q}\right), k\right)=0$ for $0<i<r(2 p-6)$;

(ii) $\mathrm{H}^{r(2 p-6)}\left(G\left(\mathbb{F}_{q}\right), k\right) \cong k$.

Proof. From Remark 6.2, for $\lambda=p \delta+u \cdot 0$ with $\delta$ in the root lattice, the following holds

$$
\operatorname{Ext}_{G}^{i}\left(V(\lambda)^{(r)}, H^{0}(\lambda)\right) \cong \begin{cases}0 & \text { if } i<2 p-3, \\ 0 & \text { if } i=r(2 p-3) \text { and } \lambda \neq(p-n)\left(\omega_{1}+\omega_{n}\right), \\ k & \text { if } i=r(2 p-3) \text { and } \lambda=(p-n)\left(\omega_{1}+\omega_{n}\right) .\end{cases}
$$

From now on assume that $\lambda=p \delta+u \cdot 0$ and that $\delta$ not in the root lattice. Our goal is to obtain results like (6.14.1) for this situation.

Assume further that $\operatorname{Ext}_{G}^{i}\left(V(\lambda)^{(r)}, H^{0}(\lambda)\right) \neq 0$. By Proposition 4.2, there exists a sequence of non-zero weights $\lambda=\gamma_{0}, \gamma_{1}, \ldots, \gamma_{r-1}, \gamma_{r}=\lambda \in X(T)_{+}$and nonnegative integers $l_{1}, l_{2}, \ldots, l_{r}$ such that

(i) $i=\sum_{j=1}^{r} l_{j}$,

(ii) $\operatorname{Ext}_{G}^{l_{j}}\left(V\left(\gamma_{j}\right)^{(1)}, H^{0}\left(\gamma_{j-1}\right)\right) \neq 0$, for $1 \leq j \leq r$, and

(iii) $\gamma_{j}=p \delta_{j}+u_{j} \cdot 0$ for some $u_{j} \in W$ and nonzero $\delta_{j} \in X(T)_{+}$.

Note that none of the $\delta_{j}$ are contained in the root lattice.

Next we apply our discussion in Section 6.3 to the pair of weights $\gamma_{j}, \gamma_{j-1}$ and obtain from 6.3 .2

$$
l_{j} \geq 2 p M_{m\left(\delta_{j}\right)}\left(\delta_{j}\right)+2 M_{m\left(\delta_{j}\right)}\left(u_{j} \cdot 0\right)-2 M_{m\left(\delta_{j-1}\right)}\left(\delta_{j-1}\right)-M_{m\left(\delta_{j-1}\right)}\left(u_{j-1} \cdot 0\right) .
$$

It follows from $\delta_{r}=\delta_{0}$ and $u_{r}=u_{0}$ that

$$
\begin{aligned}
i=\sum_{j=1}^{r} l_{j} & \geq \sum_{j=1}^{r} 2 p M_{m\left(\delta_{j}\right)}\left(\delta_{j}\right)+2 M_{m\left(\delta_{j}\right)}\left(u_{j} \cdot 0\right)-2 M_{m\left(\delta_{j-1}\right)}\left(\delta_{j-1}\right)-M_{m\left(\delta_{j-1}\right)}\left(u_{j-1} \cdot 0\right) \\
& =\sum_{j=1}^{r} 2(p-1) M_{m\left(\delta_{j}\right)}\left(\delta_{j}\right)+M_{m\left(\delta_{j}\right)}\left(u_{j} \cdot 0\right) \\
& \geq \sum_{j=1}^{r} 2(p-1) M_{m\left(\delta_{j}\right)}\left(\delta_{j}\right)+M_{m\left(\delta_{j}\right)}(-2 \rho),
\end{aligned}
$$

where the last inequality follows from Observation 6.3(f). We define

$$
d_{j}:=l_{j}+2 M_{m\left(\delta_{j-1}\right)}\left(\delta_{j-1}\right)-2 M_{m\left(\delta_{j}\right)}\left(\delta_{j}\right)-M_{m\left(\delta_{j}\right)}\left(u_{j} \cdot 0\right)+M_{m\left(\delta_{j-1}\right)}\left(u_{j-1} \cdot 0\right) .
$$

Then $i=\sum_{j=1}^{r} d_{j}$ and $d_{j} \geq 2(p-1) M_{m\left(\delta_{j}\right)}\left(\delta_{j}\right)+M_{m\left(\delta_{j}\right)}(-2 \rho)$.

In order to show vanishing up to the claimed degrees it is sufficient to show that $\sum_{j=1}^{r} d_{j} \geq$ $r(2 p-3),(r(2 p-6)$, respectively). We will actually show that strict inequalities hold in all but very few special cases. These special cases will yield statements (b)(ii) and (d)(ii) of the theorem.

According to Observation 6.3(e), we know that $M_{m\left(\delta_{j}\right)}(-2 \rho)=-N_{m\left(\delta_{j}\right)}$. Moreover, using the arguments in the proofs of Propositions 6.2, 6.3, and 6.4, one obtains the following 
ON THE VANISHING RANGES FOR THE COHOMOLOGY OF FINITE GROUPS OF LIE TYPE 3

bounds (recall that $\delta_{j}$ does not lie in the root lattice):

$$
d_{j} \geq \begin{cases}{\left[2\left(\frac{p-1}{n+1}\right)-1\right]\left(n+1-m\left(\delta_{j}\right)\right) m\left(\delta_{j}\right)} & \text { if } \delta_{j}=\omega_{m\left(\delta_{j}\right)}, \\ 2(p-1)+2(n-1)\left[\frac{p-1}{n+1}-1\right] & \text { else. }\end{cases}
$$

Note that, as observed in Section 6.4, the second expression is strictly larger than $2 p-3$ under the assumptions on $p$ and $n$. Therefore, the only way to possibly obtain a $d_{j}$ with $d_{j} \leq 2 p-3\left(2 p-6\right.$, respectively) occurs when $\delta_{j}$ is a single fundamental weight.

Case 1: $\delta_{j} \in\left\{\omega_{2}, \ldots, \omega_{n-1}\right\}, n>2$.

The expression $\left[2\left(\frac{p-1}{n+1}\right)-1\right]\left(n+1-m\left(\delta_{j}\right)\right) m\left(\delta_{j}\right)$ attains a minimum when $m\left(\delta_{j}\right)=2$ or $n-1$ (see Section 6.8). Hence,

$$
\begin{aligned}
d_{j} & \geq 2(p-1)+(2 n-6)\left(\frac{p-1}{n+1}\right)-2 n+2 \\
& \geq 2(p-1)+2(2 n-6)-2 n+2 \\
& =2(p-1)+2 n-10 .
\end{aligned}
$$

If $n \geq 5$ one obtains $d_{j} \geq 2 p-2>2 p-3$. For $n=4$ one can show that $d_{j}>2 p-3$ whenever $p>13$. We will discuss the case $n=4$ and $p \in\{11,13\}$ separately later. For $n=3$ one obtains $d_{j} \geq 2 p-6$.

Case 2: $\delta_{j}=\omega_{1}$ or $\omega_{n}$.

Here the above methods produce the lower bound

$$
d_{j} \geq 2(p-1)-2\left(\frac{p-1}{n+1}\right)-n,
$$

which is not sufficient. Other methods have to be applied. We will distinguish two cases.

Case 2.1: $\delta_{j-1}$ is a fundamental weight.

We apply Lemma 6.9 to obtain from equation (6.9.1)

$$
l_{j} \geq(p-k) n-(n+1-k) k-(n+1-s) s+\ell\left(u_{j-1}\right),
$$

where $k=\ell\left(u_{j}\right)=-M_{1}\left(u_{j} \cdot 0\right)$. By $(6.14 .3)$

$$
d_{j} \geq l_{j}+2 M_{s}\left(\omega_{s}\right)-2 M_{1}\left(\omega_{1}\right)-M_{1}\left(u_{j} \cdot 0\right)+M_{s}\left(u_{j-1} \cdot 0\right) .
$$

From Observation 2.2(B) (see Section 6.3), we know that $\ell\left(u_{j-1}\right) \geq-M_{s}\left(u_{j-1} \cdot 0\right.$ ) and clearly $M_{s}\left(\omega_{s}\right) \geq M_{1}\left(\omega_{1}\right)$. This yields

$$
d_{j} \geq(p-k) n-(n+1-k) k+k-(n+1-s) s=(p-k) n-(n-k) k-(n+1-s) s .
$$

As a function of $k$, the above attains its minimum at $k=n$. Hence

$$
d_{j} \geq(p-n) n-(n+1-s) s=2 p-3+(n-2) p-n^{2}-(n+1-s) s+3 .
$$


Using the assumption that $p \geq 2 n+3$, one obtains

$$
\begin{aligned}
d_{j} & \geq 2 p-3+(n-2)(2 n+3)-n^{2}-(n+1-s) s+3 \\
& =2 p-3+n^{2}-n-(n+1-s) s-3 .
\end{aligned}
$$

As an integer function of $s$, the above attains its minimum at

$$
s= \begin{cases}(n+1) / 2 & \text { for } n \text { odd } \\ n / 2 & \text { for } n \text { even. }\end{cases}
$$

One concludes that

$$
d_{j} \geq \begin{cases}2 p-3+\frac{3(n-1)^{2}}{4}-4 & \text { for } n \text { odd } \\ 2 p-3+\frac{3 n(n-2)}{4}-3 . & \text { for } n \text { even }\end{cases}
$$

For $n>3$ this yields $d_{j}>2 p-3$, for $n=3$ this yields $d_{j} \geq 2 p-4$, and for $n=2$ this yields $d_{j} \geq 2 p-6$.

Case 2.2: $\delta_{j-1}$ is not a fundamental weight.

Here we will show that $d_{j}+d_{j-1} \geq 2(2 p-3)$ (in the generic case). If $j=1$ we set $d_{0}=d_{r}$. Note that the following argument makes sense because $\gamma_{0}=\gamma_{r}$. Recall that none of the $\delta_{j}$ are contained in the root lattice. Therefore, (6.14.4) yields

$$
d_{j-1} \geq 2(p-1)+2(n-1)\left[\frac{p-1}{n+1}-1\right] .
$$

Adding (6.14.5) and (6.14.6) produces

$$
\begin{aligned}
d_{j}+d_{j-1} & \geq 2(2 p-3)+2+2(n-2)\left(\frac{p-1}{n+1}\right)-3 n+2 \\
& \geq 2(2 p-3)+4(n-2)-3 n+4 \\
& =2(2 p-3)+n-4 .
\end{aligned}
$$

If $n \geq 5$ one obtains $d_{j}+d_{j-1}>2(2 p-3)$. The same holds for $n=4$ and $p>11$ (since the second inequality above is in fact strict). For $n=4$ and $p=11$, one has $d_{j}+d_{j-1}=2(2 p-3)$ only if $\delta_{j-1} \in\left\{\omega_{1}+\omega_{2}, \omega_{1}+\omega_{3}, \omega_{4}+\omega_{2}, \omega_{4}+\omega_{3}\right\}$. We will discuss this case later. For all others weights we get a strict inequality. For $n=2$ and $n=3$ it follows that $d_{j}+d_{j-1} \geq 2(2 p-4)$.

Assume now that $n>3$. If $n=4$ assume in addition that $p>13$. From above, we see that $d_{j}>2 p-3$ unless $\delta_{j}=\omega_{1}$ or $\omega_{n}$ and $\delta_{j-1}$ is not a fundamental weight. If $d_{j}>2 p-3$ for each $1 \leq j \leq r$, we have

$$
i \geq \sum_{j=1}^{r} d_{j}>r(2 p-3)
$$

and vanishing for positive degrees up to $r(2 p-3)$.

Suppose now that $d_{j} \leq 2 p-3$ for some $j$. Recall that none of $\delta_{j}$ are assumed to be in the root lattice. Let $t$ be the largest such $j$ and suppose that $t>1$. Then

$$
i \geq \sum_{j=1}^{r} d_{j}=\sum_{t+1}^{r} d_{j}+d_{t}+d_{t-1}+\sum_{j=1}^{t-2} d_{j}>(r-t)(2 p-3)+2(2 p-3)+\sum_{j=1}^{t-2} d_{j} .
$$


Consider the remaining sum $\sum_{j=1}^{t-2} d_{j}$, again identify the largest $j$ with $d_{j} \leq 2 p-3$, and repeat this decomposition. Continuing in this manner the claim follows except possibly if we reach a point when the largest $j$ with $d_{j} \leq 2 p-3$ is $j=1$. So we are done if $d_{1}>2 p-3$.

Suppose now that $\delta_{1}=\omega_{1}$ or $\omega_{n}$ and $\delta_{0}$ is not a fundamental weight so that we could have $d_{1} \leq 2 p-3$. Note that since $\delta_{1}$ is a fundamental weight, $d_{2}>2 p-3$. Recall that $\delta_{0}=\delta_{r}$. Therefore, we can use the Case 2.2 argument to show that $d_{1}+d_{r}>2(2 p-3)$. Further, since $d_{2}>2 p-3$, the above argument can successfully be used to show that $\sum_{j=2}^{r-1} d_{j}>(r-2)(2 p-3)$. Hence,

$$
i \geq d_{1}+d_{r}+\sum_{j=2}^{r-1} d_{j}>2(2 p-3)+(r-2)(2 p-3)=r(2 p-3) .
$$

One concludes that (6.14.1) holds for any dominant weight $\lambda$ as long as $n \geq 5$ or $n=4$ and $p>13$.

For $n=3$ the above cases show that $i=\sum_{i=1}^{r} d_{j} \geq r(2 p-6)$ with equality only in the case that all $\delta_{j}=\omega_{2}$. Using the arguments in Section 6.11 one concludes that for weights $\mu_{1}=p \omega_{2}+w_{1} \cdot 0$ and $\mu_{2}=p \omega_{2}+w_{2} \cdot 0$

$\operatorname{Ext}_{G}^{i}\left(V\left(\mu_{2}\right)^{(1)}, H^{0}\left(\mu_{1}\right)\right)= \begin{cases}0 & \text { if } 0<i<2 p-6, \\ 0 & \text { if } i=2 p-6 \text { and not both } \mu_{1}, \mu_{2} \text { are equal to }(p-4) \omega_{2}, \\ k & \text { if } i=2 p-6 \text { and } \mu_{1}=\mu_{2}=(p-4) \omega_{2} .\end{cases}$

An argument similar to those in Sections 5.4 and 6.2 now yields

$$
\operatorname{Ext}_{G}^{i}\left(V(\lambda)^{(r)}, H^{0}(\lambda)\right)= \begin{cases}0 & \text { if } 0<i<r(2 p-6) \\ 0 & \text { if } i=r(2 p-6) \text { and } \lambda \neq(p-4) \omega_{2} \\ k & \text { if } i=r(2 p-6) \text { and } \lambda=(p-4) \omega_{2}\end{cases}
$$

If $n=2$ and 3 does not divide $p^{r}-1$ then $\operatorname{Ext}_{G}^{i}\left(V(\lambda)^{(r)}, H^{0}(\lambda)\right) \neq 0$ forces $\lambda$ and all the $\gamma_{j}$ to be in the root lattice. By Remark 6.2 one obtains (6.14.1).

If $n=2$ and 3 divides $p^{r}-1$, one concludes from the above discussion that $i=\sum_{i=1}^{r} d_{j} \geq$ $r(2 p-6)$ with equality only in the case that all $\delta_{j} \in\left\{\omega_{1}, \omega_{2}\right\}$. A direct computation using Lemma 6.9 now shows that for weights $\mu_{1}=p \omega_{r}+w_{1} \cdot 0$ and $\mu_{2}=p \omega_{s}+w_{2} \cdot 0$ with $r, s \in\{1,2\}$

$$
\operatorname{dim} \operatorname{Ext}_{G}^{i}\left(V\left(\mu_{2}\right)^{(1)}, H^{0}\left(\mu_{1}\right)\right)= \begin{cases}0 & \text { if } 0<i<2 p-6, \\ 0 & \text { if } i=2 p-6 \\ & \text { and not both } \mu_{1}, \mu_{2} \text { are in }\left\{(p-3) \omega_{i} \mid i=1,2\right\}, \\ \delta_{r s} \quad & \text { if } i=2 p-6, \text { both } \mu_{1}, \mu_{2} \in\left\{(p-3) \omega_{i} \mid i=1,2\right\} \\ & \text { and } p \equiv 1 \bmod 3, \\ 1-\delta_{r s} & \text { if } i=2 p-6, \text { both } \mu_{1}, \mu_{2} \in\left\{(p-3) \omega_{i} \mid i=1,2\right\} \\ & \text { and } p \equiv-1 \bmod 3 .\end{cases}
$$


Arguments like the ones in Sections 5.4 and 6.2 now yield

$$
\operatorname{Ext}_{G}^{i}\left(V(\lambda)^{(r)}, H^{0}(\lambda)\right)= \begin{cases}0 & \text { if } 0<i<r(2 p-6), \\ 0 & \text { if } i=r(2 p-6) \text { and } \lambda \neq(p-3) \omega_{i}, i \in\{1,2\}, \\ k & \text { if } i=r(2 p-6) \text { and } \lambda=(p-3) \omega_{i}, i \in\{1,2\}\end{cases}
$$

Note that in the case when 3 divides $p-1$ all $\delta_{j}$ are the same, while in the case that 3 divides $p+1$ the $\delta_{j}$ alternate between $\omega_{1}$ and $\omega_{2}$.

That leaves only the cases $n=4$ and $p=11$ or 13 . We will show that (6.14.1) holds in these cases. We discuss $p=13$ first. Looking at Cases 1 through 2.2 one can see that $d_{j} \leq 2 p-3$ occurs when $\delta_{j}=\omega_{2}$ or its dual weight $\omega_{3}$. We will discuss the case $\delta_{j}=\omega_{2}$. We distinguish two cases, namely, $\delta_{j-1}$ being the sum of at least two fundamental weights and $\delta_{j-1}$ being a fundamental weight. In the first situation one can use an argument similar to Case 2.2 to show that $d_{j}+d_{j-1}>2(2 p-3)$. We leave the details to the interested reader. In the second situation the weight $\delta_{j-1}=\omega_{1}$, because $p \delta_{j}-\delta_{j-1}$ has to be an element of the root lattice. In our series of estimates for $i$ and $l_{j}$ in (6.3.1), (6.3.2), and (6.14.2), we replaced $-2 M_{m\left(\delta_{j}\right)}\left(\delta_{j-1}\right)$ by $-2 M_{m\left(\delta_{j-1}\right)}\left(\delta_{j-1}\right)$. The former clearly being greater than or equal to the latter. Without this substitution one can obtain a better estimate for $d_{j}$, namely $2(p-1) M_{m\left(\delta_{j}\right)}\left(\delta_{j}\right)+M_{m\left(\delta_{j}\right)}(-2 \rho)+2 M_{m\left(\delta_{j-1}\right)}\left(\delta_{j-1}\right)-2 M_{m\left(\delta_{j}\right)}\left(\delta_{j-1}\right)$. In our special case this yields $d_{j} \geq 2 \cdot 12 \cdot 6 / 5-6+2 \cdot 4 / 5-2 \cdot 3 / 5>23$, as required.

When $n=4$ and $p=11$ we need to revisit Case 1 with $\delta_{j}=\omega_{2}$ or $\omega_{3}$. We proceed as in the $p=13$ case. If $\delta_{j-1}$ is the sum of more than one fundamental weight an argument similar to Case 2.2 will show that $d_{j}+d_{j-1}>2(2 p-3)$. If $\delta_{j-1}$ is a single fundamental weight then the prime forces $\delta_{j-1}=\delta_{j}$. Here Lemma 6.12 implies $d_{j}>2 p-3$. Details are left to the reader. The last remaining situation is when $\delta_{j}=\omega_{1}$ or $\omega_{4}$. Note that equality holds in (6.14.6) if and only if $\delta_{j-1} \in\left\{\omega_{1}+\omega_{2}, \omega_{1}+\omega_{3}, \omega_{4}+\omega_{2}, \omega_{4}+\omega_{3}\right\}$. In any of these four cases the height argument used in Case 2.1 yields $d_{j}=2 p-4$. From (6.14.6) one obtains $d_{j-1}=2 p+4$. Hence $d_{j}+d_{j-1}>2(2 p-3)$ and (6.14.1) holds for $n \geq 4$.

The weights $\lambda=(p-n) \widetilde{\alpha}$ as well as $(p-4) \omega_{2}$ for $n=3$ are the lowest non-zero dominant weights in their linkage classes. Theorem 2.8(A), (6.14.1), and (6.14.7) imply parts (a), (c) and (d). Similarly, a slight variation of Theorem 2.8(A) and (6.14.8) yield part (b).

6.15 The General Linear Group $G L_{n}\left(\mathbb{F}_{q}\right)$. For convenience we assumed throughout the paper that $G$ is a simple algebraic group. However, most results can be generalized to split reductive groups such as $G L_{n}(k)$. In particular Sections 2.3 through 2.8 and Formula (3.2.1) are valid for this larger set of groups. One can therefore argue as in Sections 6.1 and 6.2 and obtain the following:

Proposition . Suppose $n \geq 1, r \geq 1$ and $p \geq n+2$. Let $\lambda=(p-n) \tilde{\alpha}$, $\tilde{\alpha}$ being the maximal positive root. Then

(a) $\mathrm{H}^{r(2 p-3)}\left(G L_{n}(k), H^{0}(\lambda) \otimes H^{0}\left(\lambda^{*}\right)^{(1)}\right) \cong k$;

(b) $\mathrm{H}^{r(2 p-3)}\left(G L_{n}\left(\mathbb{F}_{q}\right), k\right) \neq 0$.

In [FP, Appendix] Friedlander and Parshall found the sharp bound $r(2 p-3)$ for the Borel subgroup $B\left(\mathbb{F}_{q}\right)$ of $G L_{n}\left(\mathbb{F}_{q}\right)$ for all odd $q$. Using the fact that the restriction map 
ON THE VANISHING RANGES FOR THE COHOMOLOGY OF FINITE GROUPS OF LIE TYPE

$\mathrm{H}^{i}\left(G L_{n}\left(\mathbb{F}_{q}\right), k\right) \rightarrow \mathrm{H}^{i}\left(B\left(\mathbb{F}_{q}\right), k\right)$ is injective one obtains the vanishing range of $0<i<$ $r(2 p-3)$ for the cohomology of $G L_{n}\left(\mathbb{F}_{q}\right)$. One can now combine this with the aforementioned proposition to obtain the following theorem which verifies the conjecture in Barbu $B$, Conjecture 4.11] for $p \geq n+2$.

Theorem . Suppose $n \geq 1$.

(a) If $q$ is odd, then $\mathrm{H}^{i}\left(G L_{n}\left(\mathbb{F}_{q}\right), k\right)=0$ for $0<i<r(2 p-3)$;

(b) If $p \geq n+2$, then $\mathrm{H}^{r(2 p-3)}\left(G L_{n}\left(\mathbb{F}_{q}\right), k\right) \neq 0$.

\section{REFERENCES}

[AJ] H. H. Andersen, J. C. Jantzen, Cohomology of induced representations for algebraic groups, Math. Ann., 269, (1984), 487-525.

[AM] A. Adem, R.J. Milgram, Cohomology of Finite Groups, Springer-Verlag, Berlin Heidelberg, 1994.

[B] A. Barbu, On the range of non-vanishing $p$-torsion cohomology for $G L_{n}\left(\mathbb{F}_{p}\right)$, J. Algebra, 278, (2004), 456-472.

[BNP1] C. P. Bendel, D. K. Nakano, C. Pillen, On comparing the cohomology of algebraic groups, finite Chevalley groups, and Frobenius kernels, J. Pure 85 Appl. Algebra, 163, no. 2, (2001), 119-146.

[BNP2] C. P. Bendel, D. K. Nakano, C. Pillen, Extensions for finite Chevalley groups I, Adv. Math., 183, (2004), 380-408.

[BNP3] C. P. Bendel, D. K. Nakano, C. Pillen, Extensions for finite Chevalley groups II, Trans. Amer. Math. Soc., 354, no. 11, (2002), 4421-4454.

[BNP4] C. P. Bendel, D. K. Nakano, C. Pillen, Extensions for Frobenius kernels, J. Algebra, 272, (2004), 476-511.

[BNP5] C. P. Bendel, D. K. Nakano, C. Pillen, Extensions for finite groups of Lie type: twisted groups, in Finite Groups 2003, eds. Ho et al., de Gruyter, New York, 2004, pp. 29-46.

[BNP6] C. P. Bendel, D. K. Nakano, C. Pillen, Extensions for finite groups of Lie Type II: filtrating the truncated induction functor, Cont. Math., 413, (2006), 1-23.

[BNP7] C. P. Bendel, D. K. Nakano, C. Pillen, Second cohomology groups for Frobenius kernels and related structures, Adv. Math., 209, (2007), 162-197.

[BC] W. Bosma, J. Cannon, Handbook on Magma Functions, Sydney University, 1996.

[BCP] W. Bosma, J. Cannon, C. Playhoust, The Magma Algebra System I: The User Language, J. Symbolic Computation, 3/4 no. 24, (1997), 235-265.

[Br] B. Broer, Line bundles on the cotangent bundle of the flag variety, Invent. Math., 113, (1993), $1-20$.

[C] J. F. Carlson, The cohomology of irreducible modules over $S L\left(2, p^{n}\right)$, Proc. London Math. Soc. (3), 47, (1983), 480-492.

[CLN] J.F. Carlson, Z. Lin, D.K. Nakano, Support varieties for modules over Chevalley groups and classical Lie algebras, Transactions of the AMS, 360, (2008), 1879-1906.

[F] E. M. Friedlander, Computations of $K$-theories of finite fields, Topology, 15, (1976), 87-109.

[FP] E. M. Friedlander, B. J. Parshall On the cohomology of algebraic and related finite groups, Invent. Math., 74, (1983), 85-118.

[H] H. L. Hiller, Cohomology of Chevalley groups over finite fields, J. Pure Appl. Alg., 16, (1980), 259-263.

[Hum1] J. E. Humphreys, Introduction to Lie Algebras and Representation Theory, Springer-Verlag, 1972.

[Hum2] J. E. Humphreys, Modular Representations of Finite Groups of Lie Type, Amer. Math. Soc., Providence, RI, 2003.

[J1] J. C. Jantzen, Representations of Algebraic Groups, American Mathematical Society, Mathematical Surveys and Monographs, 107, 2003.

[J2] J.C. Jantzen, Nilpotent orbits in representation theory, Lie theory, Progr. Math., 228, 1-211, Birkhuser Boston, Boston, MA, 2004 
[KLT] S. Kumar, N. Lauritzen, J. F. Thomsen, Frobenius splitting of cotangent bundles of flag varieties, Invent. Math., 136, (1999), 603-621.

[Q] D. G. Quillen, On the cohomology and K-theory of finite fields, Ann. Math., 96, (1972), 551-586.

[W] C. B. Wright, Second cohomology groups for algebraic groups and their Frobenius kernels, preprint, 2009.

Department of Mathematics, Statistics and Computer Science, University of WisconsinStout, Menomonie, WI 54751, USA

E-mail address: bendelc@uwstout.edu

Department of Mathematics, University of Georgia, Athens, GA 30602, USA

E-mail address: nakano@math.uga.edu

Department of Mathematics and Statistics, University of South Alabama, Mobile, AL 36688, USA

E-mail address: pillen@jaguar1.usouthal.edu 